\title{
Pre-therapeutic Microglia Activation and Sex Determine Therapy Effects of Chronic Immunomodulation
}

Gloria Biechele ${ }^{1}$, Tanja Blume (PhD $)^{1,2}$, Maximilian Deussing (MD) ${ }^{1}$, Benedikt Zott (MD, $\mathrm{PhD})^{3,4}$, Yuan Shi ${ }^{2}$, Xianyuan Xiang $(\mathrm{PhD})^{5}$, Nicolai Franzmeier $(\mathrm{PhD})^{6}$, Gernot Kleinberger $(\mathrm{PhD})^{7}$, Finn Peters $(\mathrm{PhD})^{2}$, Katharina Ochs ${ }^{2}$, Carola Focke ${ }^{1}$, Christian Sacher ${ }^{1}$, Karin Wind ${ }^{1}$, Claudio Schmidt ${ }^{1}$, Simon Lindner (PhD) ${ }^{1}$, Franz-Josef Gildehaus (PhD) ${ }^{1}$, Florian Eckenweber $^{1}$, Leonie Beyer (MD) ${ }^{1}$, Barbara von Ungern-Sternberg (DVM) ${ }^{1}$, Peter Bartenstein $(\mathrm{MD})^{1,8}$, Karlheinz Baumann (PhD) ${ }^{9}$, Mario M. Dorostkar (MD, PhD $)^{2,10}$, Axel Rominger $(\mathrm{MD})^{1,8,11}$, Paul Cumming (PhD) $)^{11,12}$, Michael Willem $(\mathrm{PhD})^{5}$, Helmuth Adelsberger (PhD) ${ }^{3}$, Jochen Herms $(\mathrm{MD})^{2,8,10}$, Matthias Brendel (MD) $)^{1,8}$

\footnotetext{
${ }^{1}$ Dept. of Nuclear Medicine, University Hospital of Munich, LMU Munich, Munich, Germany

${ }^{2}$ DZNE - German Center for Neurodegenerative Diseases, Munich, Germany

${ }^{3}$ Institute of Neuroscience, Technical University of Munich, Munich, Germany

${ }^{4}$ Department of Diagnostic and Interventional Neuroradiology, Klinikum rechts der Isar, Technical University of Munich, Munich, Germany

${ }^{5}$ Metabolic Biochemistry, Biomedical Center (BMC), Faculty of Medicine, Ludwig-MaximiliansUniversität München, Munich, Germany

${ }^{6}$ Institute for Stroke and Dementia Research, University Hospital of Munich, LMU Munich, Munich

Germany

${ }^{7}$ ISAR Bioscience GmbH, 82152 Planegg, Germany

${ }^{8}$ SyNergy, University of Munich, Munich, Germany

${ }^{9}$ Roche Pharma Research and Early Development, Neuroscience Discovery, Roche, Innovation Center Basel, F. Hoffmann-La Roche Ltd., Basel, Switzerland

${ }^{10}$ Center for Neuropathology and Prion Research, Ludwig-Maximilians-University of Munich, Munich, Germany

${ }^{11}$ Dept. of Nuclear Medicine, Inselspital Bern, Bern, Switzerland

${ }^{12}$ School of Psychology and Counselling, Queensland University of Technology, Brisbane, Australia
}

May 30, 2021

Running title: TSPO-PET and Sex Predict Immunomodulation Word Count:

Figures: 5

Tables: 1

Keywords: pioglitazone, TSPO-PET, App ${ }^{N L-G-F}$ mice, PS2APP mice, microglia, sex, prediction

\section{Corresponding author:}

Dr. Matthias Brendel, MHBA

Department of Nuclear Medicine

University Hospital of Munich, Marchioninistr.15, 81377 Munich, Germany

Phone: +49 (0) 89440074650

Fax: +49 (0) 89440077646

E-Mail: matthias.brendel@med.uni-muenchen.de 


\section{Abstract}

Modulation of the innate immune system is emerging as a promising therapeutic strategy against Alzheimer's disease (AD). However, determinants of a beneficial therapeutic effect are ill-understood. Thus, we investigated the potential of $18 \mathrm{kDa}$ translocator protein positron-emission-tomography (TSPO-PET) for assessment of microglial activation in mouse brain before and during chronic immunomodulation. Serial TSPO-PET was performed during five months of chronic microglia modulation by stimulation of peroxisome proliferatoractivated receptor (PPAR)- $y$ with pioglitazone in two different mouse models of $A D$ (PS2APP, App ${ }^{N L-G-F}$ ). Using mixed statistical models on longitudinal TSPO-PET data, we tested for effects of therapy and sex on treatment response. We tested correlations of baseline with longitudinal measures of TSPO-PET, and correlations between PET results with spatial learning performance and $\beta$-amyloid accumulation of individual mice. Immunohistochemistry was used to determine the molecular source of the TSPO-PET signal. Pioglitazone-treated female PS2APP and $A p p^{N L-G-F}$ mice showed attenuation of the longitudinal increases in TSPO-PET signal when compared to vehicle controls, whereas treated male $A p p^{N L-G-F}$ mice showed the opposite effect. Baseline TSPO-PET strongly predicted changes in microglial activation in treated mice $(R=-0.874, p<0.0001)$ but not in vehicle controls $(R=-0.356, p=0.081)$. Reduced TSPO-PET signal upon treatment was associated with better spatial learning and higher fibrillar $\beta$-amyloid accumulation. Immunohistochemistry confirmed activated microglia to be the source of the TSPO-PET signal $(R=0.952, p<0.0001)$. TSPO-PET represents a sensitive biomarker for monitoring of immunomodulation and closely reflects activated microglia. Pre-therapeutic assessment of baseline microglial activation and sex are strong predictors of individual immunomodulation effects and could serve for responder stratification. 


\section{Introduction}

Neuroinflammation is now recognized as an inherent part of the Alzheimer's disease (AD) pathology ${ }^{1}$. The key players of neuroinflammation in $A D$ are activated microglia and astrocytes ${ }^{2}$. Although it is still unclear if beneficial or detrimental effects of neuroinflammation dominate in the (patho)physiology of $A D$, there is considerable interest in integrating the modulation of neuroinflammation into novel treatment strategies against $A D^{3}$. Preclinical studies showed that immunomodulation by peroxisome proliferator-activated receptor (PPAR)- $y$ using the antidiabetic compound pioglitazone rescues neuronal spine density ${ }^{4}$ and spatial learning performance ${ }^{5}$ in mouse AD models. However, a large human trial with pioglitazone in mild cognitive impairment due to $A D$ was terminated after an interim analysis showing lack of efficacy ${ }^{6}$. Hence, the discrepancies between beneficial effects in preclinical studies and lacking efficacy in humans deserve detailed inquiry, with the objective of uncovering the salient factors accounting for the failure of PPARy stimulation in clinical translation.

TSPO-PET is increasingly used to monitor therapy-related changes of microglial activation in humans ${ }^{7}$ and rodent models ${ }^{8}$. In this regard, the TSPO ligand ${ }^{18} \mathrm{~F}-\mathrm{GE} 180$ is proven effective for robust imaging of microglial activation in a mouse model of amyloidosis, and the normalization of TSPO binding upon treatment with neurotrophin receptor ligand that ameliorates hyperphosphorylation and misfolding of tau and rescues the consequent neurite degeneration ${ }^{9}$. Our previous data revealed excellent agreement between ${ }^{18} \mathrm{~F}$-GE180 PET quantitation and immunohistochemistry of microglial markers ${ }^{10,11}$, thus indicating its potential to access and predict PPARy stimulation effects in vivo.

Therefore, we aimed to test the hypothesis that TSPO-PET with ${ }^{18} \mathrm{~F}-\mathrm{GE} 180$ is a suitable tool for monitoring anti-neuroinflammatory responses to chronic immunomodulation in AD mouse models. We furthermore tested the hypothesis that microglial activation by TSPO-PET predicts therapy related changes and outcome parameters. Furthermore, we tested for effects of mouse sex on immunomodulation. Finally, we used immunohistochemistry to validate in vivo PET findings and to confirm the cellular source of TSPO-PET signal alterations.

\section{Results}

\section{TSPO-PET detects altered microglia activation during chronic PPARy stimulation}

First, we investigated whether effects of chronic PPARY stimulation can be detected by TSPO PET in PS2APP mice and wild-type controls. Vehicle-treated PS2APP mice showed a strong increase over time of the TSPO-PET signal when compared to vehicle-treated wild- 
type mice between eight and 13 months of age, with a peak at 11.5 months $(+52-67 \%$, all time-points: $p<0.0001$, Figure 1). The pre-therapeutic baseline TSPO-PET signal did not significantly differ between PS2APP mice with and without pioglitazone treatment $\left(S_{U} V_{H}\right.$ : $0.24 \pm 0.05$ vs. $0.26 \pm 0.01, p=0.647$ ). However, PS2APP mice with pioglitazone treatment had a lower TSPO-PET signal at $9.5(-13 \%, p=0.0027), 11.5(-17 \%, p=0.0046)$, and 13.0 ($13 \%, p=0.0071)$ months of age when compared to age-matched vehicle-treated PS2APP mice (Figure 1). Linear mixed models revealed a main effect of treatment group on TSPOPET across time-points ( $b / S E=-0.036 / 0.006, T=5.405, p<0.0001)$, controlling for age (i.e. quadratic effect) and random intercept. Individual PS2APP mice indicated a heterogeneous pharmacotherapy-related change in the TSPO-PET signal, which was already conspicuous during the first six weeks of treatment (range of change: $-35 \%$ to $+86 \%$ ). Pioglitazone-treated wild-type mice manifested a slight decrease of the TSPO-PET signal after six weeks of treatment when compared to vehicle-treated wild-type mice $(-12 \%, p=0.013)$, and no such differences at the other time points. Taken together, we found strong differences of TSPO expression in vivo between treated and non-treated animals, suggesting the feasibility of TSPO-PET to monitor effects of PPARy stimulation.

A
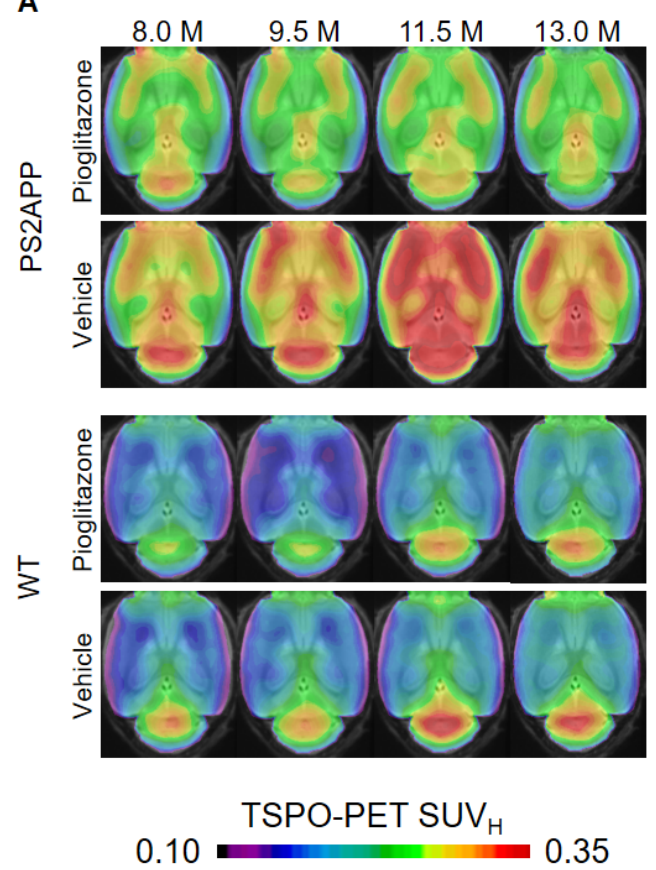

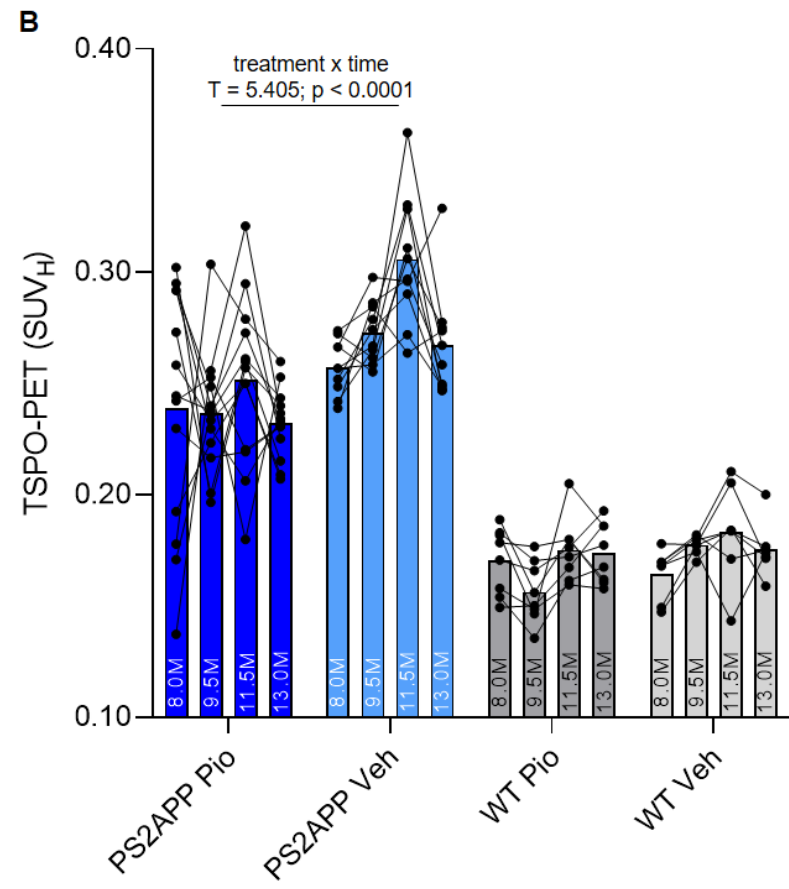

Figure 1 - TSPO-PET monitoring of chronic pioglitazone treatment in PS2APP and wild-type (WT) mice. (A) Axial images show group levels of the ${ }^{18} \mathrm{~F}$-GE180 TSPO-PET signal (myocardium scaled standardized uptake value, $S U V_{H}$ ) at different ages in treatment and vehicle groups, projected upon a standard MRI anatomic template. Baseline scans were performed prior to treatment initiation. (B) Individual time courses of the cortical TSPO-PET signal during the treatment period. Pio = pioglitazone treatment, $V$ eh $=$ vehicle treatment. Statistics derive from a linear mixed model. PS2APP pioglitazone $n=13$, PS2APP vehicle $n=10$, WT pioglitazone $n=8$, WT vehicle $n=7$. 


\section{Chronic PPARy stimulation changes microglial activation independent of APP overexpression but dependent on sex}

Next, we tested whether previously observed sex differences in TSPO expression in mouse brain ${ }^{12}$ have an impact on the responses to PPARy pharmacological stimulation. To this end, we used the novel APP knock-in model $A p p^{N L-G-F 13}$ mice and performed longitudinal TSPO$P E T$ imaging during chronic pioglitazone treatment in groups of female and male mice. Furthermore, we tested whether these mice showed effects of PPARy stimulation on the TSPO-PET signal in the absence of APP overexpression. We observed sex-specific elevation of the TSPO-PET signal in vehicle-treated female $A p p^{N L-G-F}$ mice when compared to males aged $7.5(+18 \%, p=0.017)$ and 10 months of age $(+25 \%, p=0.0007$; sex $x$ time interaction: $b / S E=-0.100 / 0.030, T=-3.273, p=0.0003$, linear mixed model controlling for random intercept). Female $A p p^{N L-G-F}$ mice with pioglitazone treatment showed normalization of the TSPO-PET signal increase compared to vehicle-treated female $A p p^{N L-G-F}$ mice aged from five to ten months (Figure 2). This resulted from decreased TSPO-PET signal in pioglitazone-treated female $A p p^{N L-G-F}$ mice aged $7.5(-15 \%, p=0.030)$ and 10 months $(-21 \%$, $\mathrm{p}=0.0053$ ) when compared to vehicle-treated female $A p p^{N L-G-F}$ mice (treatment $\mathrm{x}$ time interaction: $b / S E=0.114 / 0.030, T=3.801, p=0.0009$, linear mixed model controlling for random intercept, Figure 2). On the other hand, male $A p p^{N L-G-F}$ mice with pioglitazone treatment tended to show an exaggerated increase of the TSPO-PET signal from five to ten months of age when compared to vehicle treated male $A p p^{N L-G-F}$ mice $(+12 \%$ vs. $+2 \%$, treatment $x$ time interaction: $b / S E=-0.041 / 0.022, T=-1.862, p=0.072)$. Wild-type mice did not show differences in TSPO-PET signal between treated or non-treated animals for this time span. In summary, sex strongly influenced the magnitude of microglial response to amyloidosis, with distinctly opposite effects in female and male mice. APP overexpression at baseline was not a significant determinant of effects of PPARy stimulation on TSPO expression. 
A
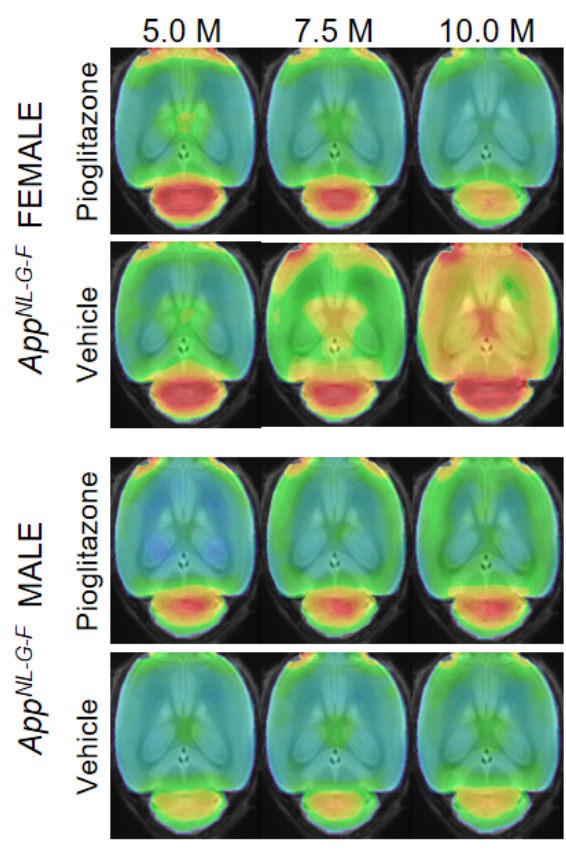

$0.10 \stackrel{\text { TSPO-PET SUV }}{\mathrm{H}} \mathbf{0 . 3 0}$
B

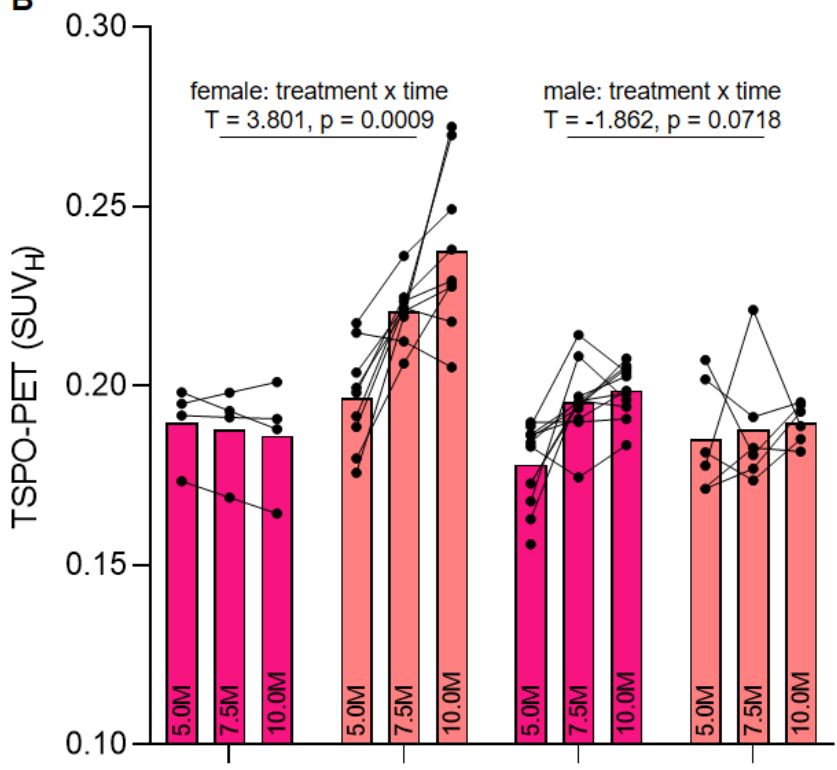

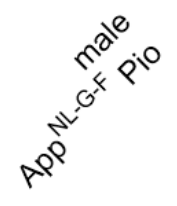

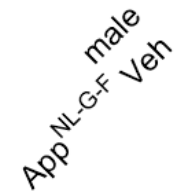

Figure $2-{ }^{18} \mathrm{~F}$-GE180 TSPO-PET monitoring of effects of chronic pioglitazone treatment in female and male $A p p^{N L-G-F}$ mice. (A) Axial images show group means of the TSPO-PET signal (myocardium scaled standardized uptake value, $\mathrm{SUV}_{\mathrm{H}}$ ) at different ages in treatment and vehicle groups, projected upon a standard MRI template. Baseline scans were performed prior to therapy initiation. (B) Individual time courses of the cortical TSPO-PET signal during the pharmacological treatment period. Statistics derive from a linear mixed model. Female $A p p^{N L-G-F}$ pioglitazone $n=4$, female $A p p^{N L-G-F}$ vehicle $\mathrm{n}=9$, male $A p p^{N L-G-F}$ pioglitazone $\mathrm{n}=11$, male $A p p^{N L-G-F}$ vehicle $\mathrm{n}=6$. Pio $=$ pioglitazone treatment, $V$ eh $=$ vehicle treatment.

\section{Baseline TSPO-PET predicts treatment associated changes in microglial activation during chronic PPARy stimulation}

Given the observed heterogeneity of changes in TSPO-PET after induction of PPARY stimulation, we asked if TSPO-PET at baseline serves to predict the individual longitudinal changes in microglial activation upon treatment. Strikingly, we observed a strong negative association between baseline TSPO-PET and subsequent changes in the TSPO-PET signal across pioglitazone treated animals $(R=-0.874, p<0.001$, Figure 3A, B), suggesting that mice with high microglial activation at baseline respond stronger to PPARY stimulation. Importantly, this association was also present in independent cohorts of PS2APP mice ( $R=$ $0.964, p<0.0001)$ and $A p p^{N L-G-F}$ mice $(R=-0.680, p=0.0053)$ mice with chronic pioglitazone treatment. On the other hand, there was only a trend towards a negative association between the baseline TSPO-PET signal and subsequent TSPO-PET changes in vehicle treated animals $(R=-0.356, p=0.081)$. The association between pre-therapeutic TSPO-PET 
results and changes in microglial activation of pioglitazone treated mice was observed across all brain regions, with the strongest differences relative to vehicle treated mice in neocortical areas, hippocampus, striatum, and thalamus (Figure 3C, D; Table 1). Several subcortical regions also showed a significant negative association between the baseline TSPO-PET signal and changes in microglial activation in the vehicle cohort (Table 1), congruent with the observation that microglial activation at baseline per se has a predictive value for longitudinal alterations of microglial activity and spatial learning performance ${ }^{14,15}$. In summary, our data indicate a strong predictive value of a baseline biomarker of microglial activation for the effect of immunomodulatory therapy.

A

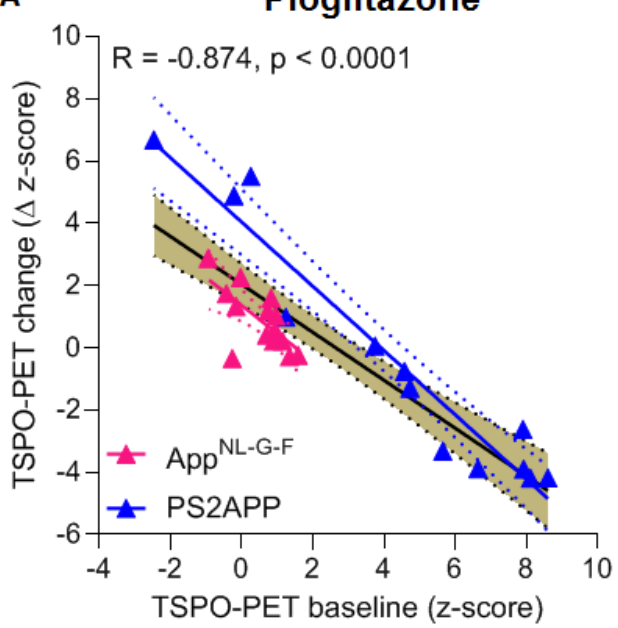

B

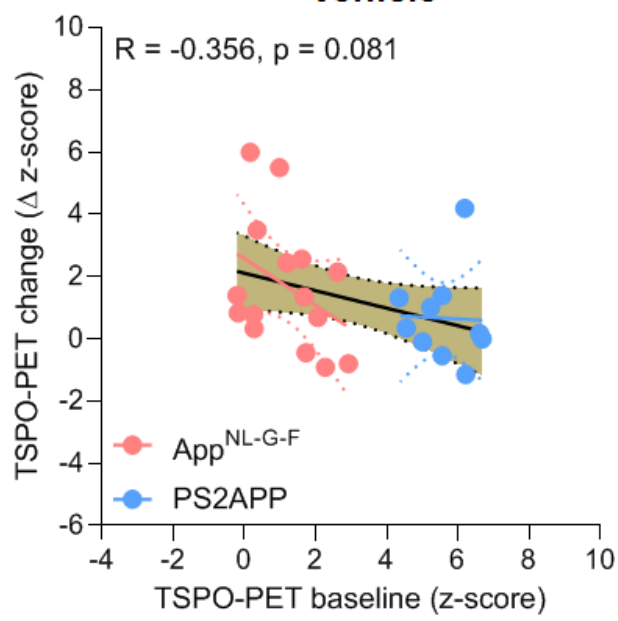

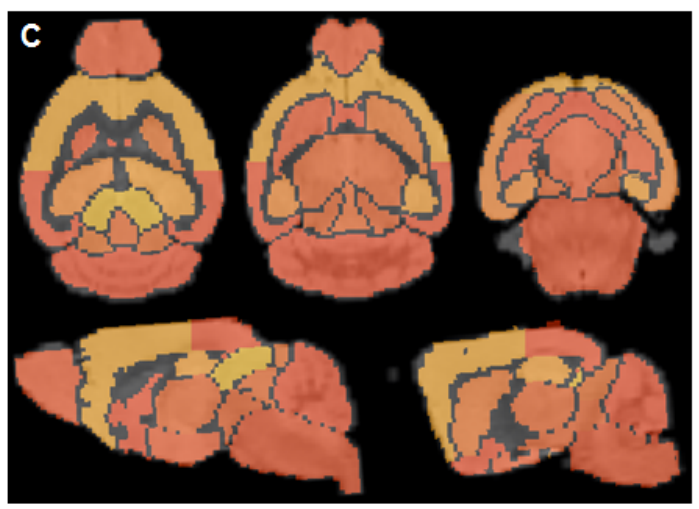
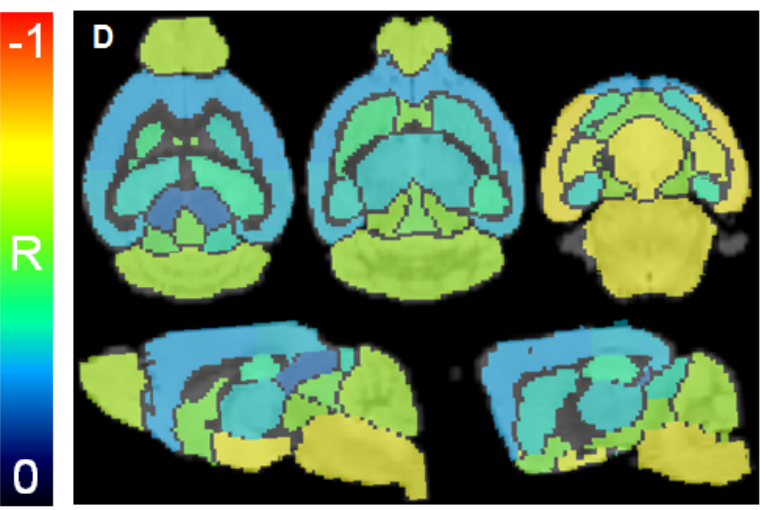

Figure 3 - Prediction of changes in microglial activity by the ${ }^{18} \mathrm{~F}$-GE180 TSPO-PET baseline examination. (A, B) Correlation analysis between the TSPO-PET z-score at baseline and the change of the TSPO-PET Z-score (baseline to last follow-up) in $A p p^{N L-G-F}$ and PS2APP mice with pioglitazone treatment $(\mathbf{A})$ and vehicle controls $(\mathbf{B})$. Correlations are illustrated for the combination of both mouse models (gold) and in separate analyses of $A p p^{N L-G-F}$ (red) and PS2APP (blue) mice. (C, D) Multiregional analysis of the correlation between the TSPO-PET z-score at baseline and the change of the TSPO-PET z-score (baseline to last follow-up). Reprojected coefficients of correlation (R) are illustrated in axial and sagittal slices projected upon a standard MRI template. App $p^{N L-G-F}$ and PS2APP mice were analyzed together for the pioglitazone treatment group (C) and vehicle controls (D). Levels of significance per region after false discovery rate correction for multiple comparisons are provided in Table 1. 


\begin{tabular}{|c|c|c|c|c|c|}
\hline \multirow[t]{2}{*}{ Region } & \multicolumn{2}{|c|}{ Pioglitazone } & \multicolumn{2}{|l|}{ Vehicle } & \multirow{2}{*}{$\begin{array}{l}\text { Contrast } \\
\Delta R\end{array}$} \\
\hline & $\mathrm{R}$ & $\begin{array}{l}\text { p-value (FDR- } \\
\text { corrected) }\end{array}$ & $\mathrm{R}$ & $\begin{array}{l}\text { p-value (FDR- } \\
\text { corrected) }\end{array}$ & \\
\hline Striatum R & -0.941 & $4.9 \mathrm{e}-13^{\star \star \star}$ & -0.427 & 0.12 & 0.514 \\
\hline Striatum L & -0.899 & $1.0 \mathrm{e}-10^{* * *}$ & -0.342 & 0.12 & 0.557 \\
\hline Hippocampus R & -0.879 & $8.6 e-10^{\star \star \star}$ & -0.344 & 0.12 & 0.535 \\
\hline Hippocampus L & -0.854 & $8.6 \mathrm{e}-09^{\star \star \star}$ & -0.388 & 0.083 & 0.466 \\
\hline Thalamus & -0.920 & $7.8 \mathrm{e}-12^{\star \star \star}$ & -0.326 & 0.13 & 0.594 \\
\hline Cerebellum & -0.952 & $1.4 \mathrm{e}-13^{\star \star \star}$ & -0.584 & $0.0066^{\star *}$ & 0.368 \\
\hline Basal forebrain \& septum & -0.949 & $1.4 \mathrm{e}-13^{\star * \star}$ & -0.516 & $0.017^{*}$ & 0.433 \\
\hline Hypothalamus & -0.936 & $8.6 \mathrm{e}-13^{\star \star \star}$ & -0.705 & $0.0009^{\star \star \star}$ & 0.231 \\
\hline Amygdala $\mathrm{R}$ & -0.935 & $8.6 \mathrm{e}-13^{\star * \star}$ & -0.665 & $0.0012^{\star *}$ & 0.270 \\
\hline Amygdala L & -0.935 & $9.0 \mathrm{e}-13^{\star \star \star}$ & -0.666 & $0.0015^{\star *}$ & 0.269 \\
\hline Brainstem & -0.943 & $1.4 \mathrm{e}-12^{\star \star \star}$ & -0.691 & $0.0009^{\star \star \star}$ & 0.252 \\
\hline Central grey & -0.914 & $1.8 \mathrm{e}-11^{\star \star \star}$ & -0.505 & $0.019^{*}$ & 0.409 \\
\hline Superior colliculi & -0.804 & $2.6 \mathrm{e}-07^{\star \star \star}$ & -0.199 & 0.34 & 0.605 \\
\hline Olfactory bulb & -0.938 & $7.5 e-13^{\star \star \star}$ & -0.603 & $0.0050^{\star \star}$ & 0.335 \\
\hline Midbrain R & -0.919 & $9.5 \mathrm{e}-12^{\star \star \star}$ & -0.558 & $0.0099^{* *}$ & 0.361 \\
\hline Midbrain L & -0.922 & $6.4 \mathrm{e}-12^{\star * *}$ & -0.541 & $0.012^{*}$ & 0.381 \\
\hline Inferior colliculus $\mathrm{R}$ & -0.900 & $3.0 \mathrm{e}-11^{* \star *}$ & -0.486 & 0.11 & 0.414 \\
\hline Inferior colliculus L & -0.910 & $9.7 e-11^{\star \star *}$ & -0.357 & $0.024^{*}$ & 0.553 \\
\hline Piriform/entorhinal cortex & -0.883 & $6.2 \mathrm{e}-10^{\star \star \star}$ & -0.742 & $0.0005^{\star \star \star}$ & 0.141 \\
\hline Auditory/visual cortex & -0.947 & $1.8 \mathrm{e}-13^{\star \star \star}$ & -0.306 & 0.15 & 0.641 \\
\hline Motor/sensory cortex & -0.836 & $3.2 \mathrm{e}-08^{\star \star \star}$ & -0.283 & 0.18 & 0.553 \\
\hline
\end{tabular}

Table 1 - Multi-region analysis of baseline prediction of longitudinal microglial activation by baseline TSPO-PET. Person's correlation coefficients $(R)$ were calculated between baseline TSPO-PET (zscore) and the change in TSPO-PET ( $\triangle$ z-score) during the five months treatment period in pioglitazone and vehicle treated $A p p^{N L-G-F}$ and PS2APP mice. P-values were adjusted for multiple comparisons by false discovery rate correction. ${ }^{*} \mathrm{p}<0.05 ;{ }^{* *} \mathrm{p}<0.01 ;{ }^{* * *} \mathrm{p}<0.001$

\section{PPARy stimulation induced changes of microglial activation predict spatial learning performance and aggregation of fibrillar $A \beta$}

Next, we asked if altered TSPO expression during chronic pioglitazone treatment has associations with known determinants of therapeutic effects. To this end, we correlated the rate of change in the TSPO-PET signal during the treatment period with the individual spatial learning impairment and changes in fibrillary $A \beta$ pathology measured post mortem. Better spatial learning was associated with an attenuated increase of the TSPO-PET signal during five months of PPARy stimulation in PS2APP mice $(R=-0.733, p=0.0043$, Figure 4A, B), but the association did not reach statistical significance in $A p p^{N L-G-F}$ mice $(R=-0.349, p=$ 0.221 , Figure 4C, D). The observed effect in PS2APP mice was treatment-specific, since 
there was no association between altered TSPO expression and spatial learning in vehicle treated mice $(R=-0.032, p=0.991$, Figure $4 B)$. Our dedicated analysis of $A \beta$ species during chronic PPARy stimulation in this same cohort ${ }^{16}$ revealed a greater increase in fibrillar $A \beta$, which is the major source of the A $\beta$-PET signal ${ }^{17}$, in both treated mouse models compared to their vehicle controls, which reflected a shift of $A \beta$ plaques towards a more fibrillary composition. Meanwhile, the non-fibrillar proportion of plaques decreased upon the treatment, as is reported elsewhere ${ }^{16}$. A low area under the curve (AUC) of TSPO-PET signal during the recording period was associated with a higher rate of change of fibrillar $A \beta$ in pioglitazone-treated PS2APP $\left(R=-0.600, p=0.030\right.$, Figure 4E, F) and $A p p^{N L-G-F}$ mice $(R$ $=-0.553, p=0.040$, Figure 4G, H). Vehicle controls of both models did not show significant associations between the TSPO-PET AUC and changes in fibrillary A $\beta$ pathology. Thus, imaging results for our treatment paradigm confirmed that higher microglial activation is associated with a slower rate of $A \beta$ accumulation, suggesting a net protective effect ${ }^{18}$.
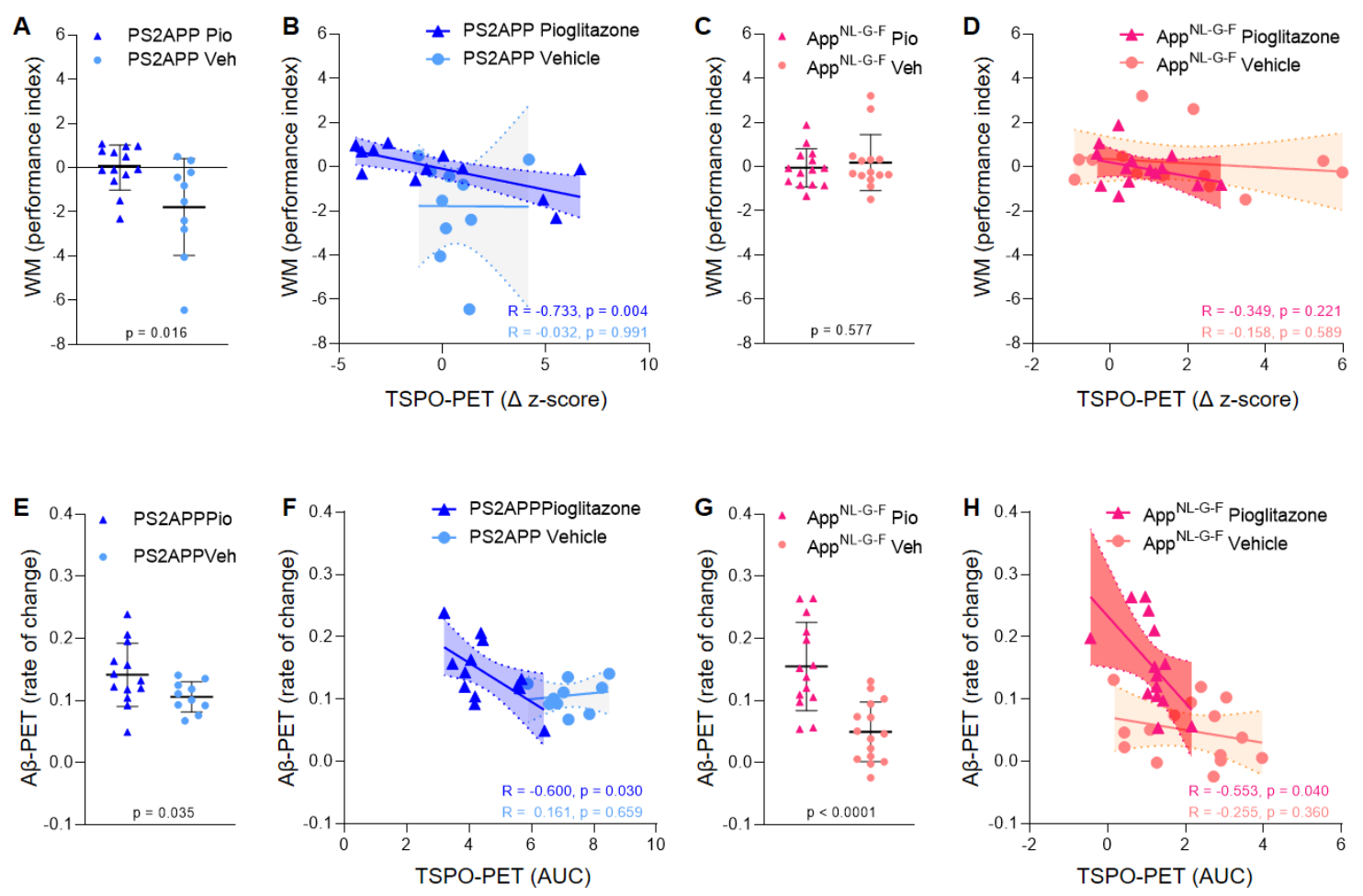

Figure 4 - Associations of ${ }^{18} \mathrm{~F}$-GE180 TSPO-PET findings with spatial learning performance and $A \beta$ accumulation. (A) Water maze (WM) performance index (principal component analysis, PCA, higher score means better performance) in the comparison of PS2APP mice after five months pioglitazone treatment and their vehicle controls. (B) Correlation between the longitudinal change of TSPO-PET in PS2APP mice and the water maze performance index. (C) Water maze performance index in the comparison of $A p p^{N L-G-F}$ mice after five months pioglitazone treatment and their vehicle controls. (D) Correlation between the longitudinal change of TSPO-PET in $A p p^{N L-G-F}$ mice and the water maze performance index. (E) A $\beta$-PET rate of change ( $\triangle$ SUVR) in the comparison of PS2APP mice after five months pioglitazone treatment and their vehicle controls ${ }^{16}$. (F) Correlation between the TSPO-PET

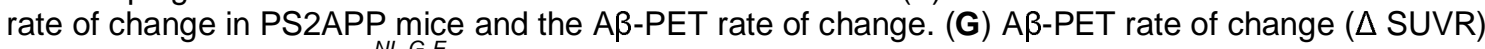
in the comparison of $A p p^{N L-G-F}$ mice after five months pioglitazone treatment and their vehicle controls 16. (H) Correlation between the TSPO-PET rate of change in $A p p^{N L-G-F}$ mice and the AB-PET rate of 
change. P-values of the group comparisons derive from an unpaired two-tailed t-test. R- and P-values of the correlation analyses derive from a Pearson correlation. PS2APP pioglitazone $n=13$, PS2APP vehicle $\mathrm{n}=10, A p p^{N L-G-F}$ pioglitazone $\mathrm{n}=15, A p p^{N L-G-F}$ vehicle $\mathrm{n}=15 . \mathrm{AUC}=$ area under the curve.

\section{${ }^{18}$ F-GE180 TSPO-PET signal reflects activated microglia}

Finally, we set about to elucidate the molecular source of the TSPO-PET signal. Earlier studies have already validated in vivo TSPO-PET as a microglial marker relative to immunohistochemistry ex vivo ${ }^{10,11}$ and we have elsewhere demonstrated that PPARyrelated modulation of microglia can be detected by terminal immunohistochemistry in these mouse models ${ }^{16}$. However, the molecular and cellular correlates of altered TSPO expression during pharmacological PPARy stimulation remained unclear. To establish this relationship, we performed an immunohistochemical validation of TSPO-PET in subpopulations of all study groups using antibodies against a general marker of microglia (Iba-1) and a specific marker of microglial activation (CD68).

Iba-1 $(R=0.790, p<0.0001$, Figure 5A) and CD68 $(R=0.952, p<0.0001$, Figure 5B) immunohistochemistry results correlated highly with TSPO-PET binding in vivo. Importantly, we saw a stronger association between TSPO-PET with CD68 labelling, which we attribute to the lesser differentiation of Iba-1 immunohistochemistry for treated $A p p^{N L-G-F}$ and PS2APP. Indeed, Iba-1 immunohistochemistry did not differentiate between treated $A p p^{N L-G-F}$ and treated PS2APP mice. The lacking differentiation of pioglitazone-treated PS2APP and App ${ }^{N L-}$ $G-F$ mice by lba-1 immunohistochemistry was also discernible at the individual mouse level (Figure 5C). This indicated that PPARy stimulation specifically reduced the activation status of microglia and that TSPO-PET predominantly tracked activated microglia. 
A

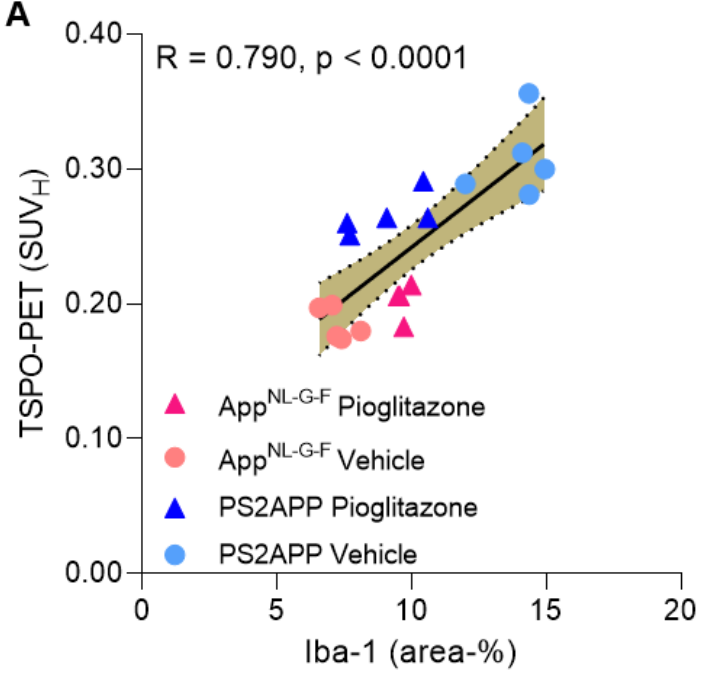

B

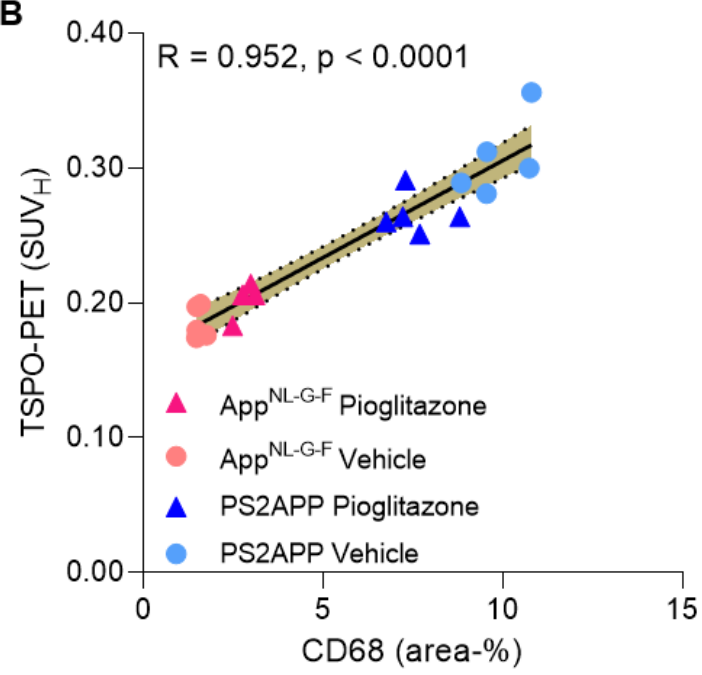

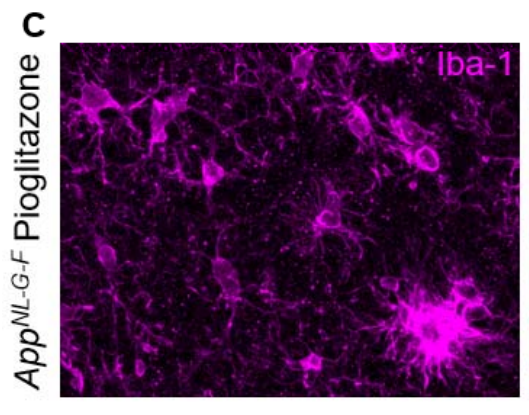
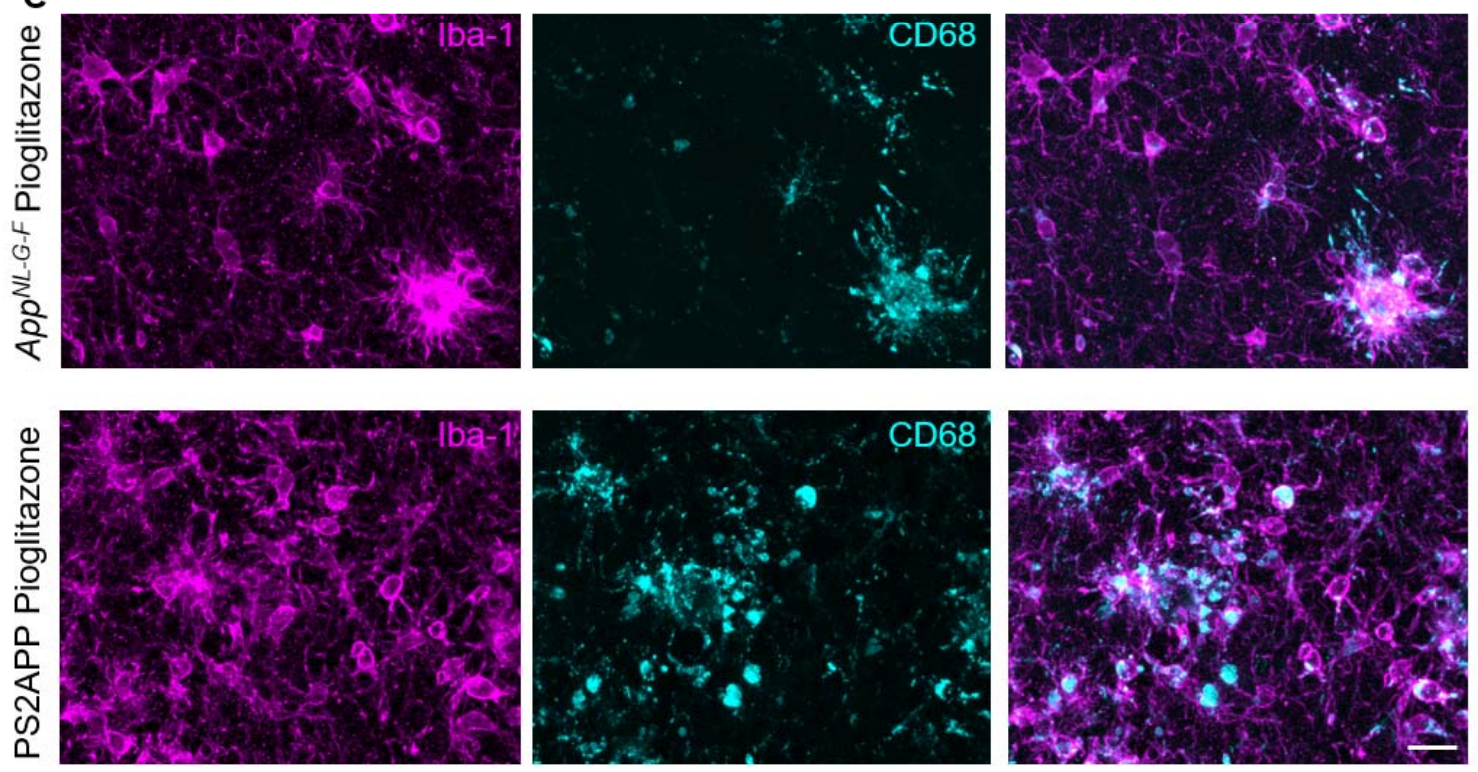

Figure 5 - (A, B) Correlation analysis between immunohistochemistry markers of microglial activation and ${ }^{18} \mathrm{~F}-\mathrm{GE} 180$ TSPO-PET at the terminal time-point. $A p p^{N L-G-F}$ pioglitazone $\mathrm{n}=4, \operatorname{App}^{N L-G-F} \mathrm{n}=5$, PS2APP pioglitazone $n=5$, PS2APP vehicle $n=5$. Error bands represent the $95 \%$ confidence intervals. $\mathrm{SUV}_{\mathrm{H}}=$ standardized uptake value including myocardium correction. $\mathrm{R}=$ Pearson's coefficient of correlation. (C) Representative immunohistochemistry images of treated $A p p^{N L-G-F}$ and PS2APP mice, indicating a similar Iba-1 area coverage but a higher CD68 area coverage of PS2APP mice compared to $A p p^{N L-G-F}$. The differentiation by CD68 fitted to corresponding TSPO-PET images, clearly showing an elevated TSPO-PET signal in PS2APP mice compared to $A p p^{N L-G-F}$. Scale bar $=20 \mu \mathrm{m}$

\section{Discussion}

In this longitudinal study, we investigated serial TSPO-PET imaging as a tool for monitoring of chronic immunomodulation in two distinct mouse models of amyloidosis. Here, PET with the TSPO ligand ${ }^{18} \mathrm{~F}$-GE180 sensitively detected changes of microglial activity upon PPARY stimulation. Furthermore, we discovered an important sex difference in treatment response. Pre-therapeutic TSPO-PET measures supported a prediction of treatment responses across 
mouse models and sexes, thus indicating that individual differences in baseline TSPO expression set the stage for effects of immunomodulation. Immunohistochemistry confirmed that TSPO-PET specifically measured activated microglia in the present models.

Our results prove that PET with the TSPO ligand ${ }^{18} \mathrm{~F}-\mathrm{GE}-180$ can sensitively monitor pioglitazone-induced changes of microglial activity during chronic treatment of AD-model mice. This finding is important given that an earlier PET study using the less avid TSPO ligand ${ }^{11} \mathrm{C}-(R)-\mathrm{PK} 11195$ failed to detect treatment-induced changes in the TSPO-PET signal during chronic pioglitazone administration in APPPS1 mice ${ }^{19}$. Nonetheless, the ${ }^{11} \mathrm{C}-(R)$ PK11195 methodology was sufficiently sensitive to detect microglial activation in transgenic versus wild-type mice. We note that a head-to-head comparison with an equal treatment setting would be required to draw robust conclusions on the superiority of one tracer over the other. However, earlier studies support our present findings of excellent sensitivity for ${ }^{18} \mathrm{~F}$ GE-180 TSPO-PET, since the tracer outperformed ${ }^{11} \mathrm{C}-(\mathrm{R})-\mathrm{PK} 11195$ in a preclinical head-tohead comparison after lipopolysaccharide challenge ${ }^{20}$ and revealed higher specific binding in vivo when compared to ${ }^{11} \mathrm{C}-\mathrm{PBR} 23$ in a human blocking study ${ }^{21,}{ }^{22}$. Importantly, we successfully measured treatment effects on microglial activation by TSPO-PET in two distinct $A \beta$ mouse models. Here, our use of $A p p^{N L-G-F}$ mice ${ }^{13}$ provided evidence that TSPO expression is altered by PPARy stimulation in mice without overexpression of APP. In line with our data, ${ }^{18} \mathrm{~F}$-GE-180 PET also enabled the detection of reduced microglial activation during neurotrophin receptor modulation by $\mathrm{LM} 11 \mathrm{~A}-31^{9}{ }^{9}$. In brief, our PET monitoring of pharmacological PPARY stimulation clarified that the direct modulation of microglial activity can be captured in vivo.

The main finding of our study is that pre-therapeutic and serial TSPO-PET recordings in our chronic pioglitazone treatment paradigm allowed predicting individual treatment responses. First, mice with high microglial activation at baseline showed stronger treatment effects, which was underpinned by a strong association between high baseline TSPO-PET quantitation and slower increase rates of TSPO-PET signal during the five months of PPARY stimulation. Thus, TSPO imaging of microglial activation can serve as a powerful translational tool ${ }^{23}$, allowing for individual predictive response stratification before or during immunomodulation in the context of precision medicine. Second, the magnitude of microglial activation during the treatment period had close associations with changes in fibrillar $A \beta$ pathology of both models and with spatial learning performance of PS2APP mice. The present finding of stronger increases of fibrillar $A \beta$ in mice with low baseline microglial activity is entirely in line with our translational study in mice with amyloidosis and AD patients ${ }^{18}$. Thus, the present results strengthen the hypothesis that activated microglia mediate the clearance of excess fibrillar $A \beta$. Interpretation of the observed association between low 
microglial activation and better spatial learning performance calls for some subtlety. Although a sufficient microglial response seems important to maintain brain function in therapy-naive $A D$ model mice ${ }^{15,24}$, the suppression of microglial activation by PPARy stimulation was directly correlated with better spatial learning performance in the current study. Thus, we suppose that PPARy stimulation shifted the already activated microglia (i.e., in mice with high TSPO-PET levels at baseline) towards a more pronounced neuroprotective function. Proving this conjecture might call for a more rigorous discrimination of the M1/M2 phenotypic characteristics. Nonetheless, present data definitely substantiate that microglia play a major role in the histological and behavioral consequences of cerebral amyloidosis in mice.

Interestingly, we observed a pronounced sex effect on the pioglitazone treatment response in $A p p^{N L-G-F}$ mice. Vehicle-treated female $A p p^{N L-G-F}$ mice showed the previously reported stronger increase of TSPO expression when compared to their male littermates ${ }^{12}$, but PPARy stimulation rectified this increase in females while exacerbating the course of neuroinflammation in males. This finding is of remarkable translation significance, since some pioglitazone studies used only female mice ${ }^{25}$ or did not declare the sex of mice ${ }^{26}$. Thus, potential sex effects of PPARy stimulation might have been missed in these studies. Furthermore, this sex effect merits attention consideration in planning human studies, since levels of sex hormones could impact upon microglial modulation ${ }^{27}$. On the other hand, our parallel detailed analysis of amyloid aggregation during chronic PPARy stimulation in this cohort did not show relevant sex differences in the rate of increase in A PET signal ${ }^{16}$. Still, this fits with our previously reported dependency of the A $\beta$-PET rate of change on microglial activity in $A D$ model mice ${ }^{18}$, since the present pioglitazone treatment in male and female $A p p^{N L-G-F}$ mice resulted in similar microglia activation levels at the end of the study.

We initiated PPARy therapy at ages manifesting an early phase of limited fibrillar amyloidosis in both mouse models, thus emulating an early but detectable stage of the human $A D$ continuum ${ }^{28}$. In consideration of emerging plasma biomarkers for $A D$ pathology ${ }^{29}$, novel treatments for $A D$ shall likely be initiated at a comparable disease stage in future clinical studies. Thus, we focused our intervention monitoring on the phase of amyloid aggregation, which revealed the greatest therapeutic response in AD model mice with a seemingly more aggressive microglial activation during early amyloid build-up. Thus, insofar as PET, cerebrospinal fluid, or plasma biomarkers of microglial activation could serve for treatment stratification in early $A D$ with positive $A \beta$-status, we foresee opportunities emerging for personalized precision medicine. The major drawback of current mouse $A D$ models is the missing conversion of a sole $A \beta$-positive stage $(A+T-)$ to combined $A \beta /$ tau-positivity $(A+T+)$. Although the recent literature describes novel combinations of $A \beta /$ tau gene modification ${ }^{30,31}$, these models still do not present a breakthrough in better mimicking human $A D$. Conceivably, cortical tau seeding in an $A \beta$ mouse model might yield a more AD-like model of 
tauopathy, but such models are not yet ready for large scaled testing of drugs ${ }^{32}$. Thus, we note as a limitation of the present study that we were unable to investigate effects of pioglitazone on conversion to tau-positivity or during subsequent tau spreading. As a consequence, we cannot predict the efficacy of chronic PPARy stimulation on the tauopathy encountered at late stages of $A D$.

The molecular sources of the TSPO-PET signal in neurodegenerative diseases remained to be fully elucidated ${ }^{22}$. We undertook a correlation analysis between TSPO-PET and immunohistochemistry endpoints in heterogeneous samples of two mouse models, factoring for age, sex, and presence or absence of immunomodulation. Here, we found that the activated microglial marker CD68 proved to have a much better correlation with TSPO-PET signal. In contrast, Iba-1 immunohistochemistry did not distinguish between PS2APP and $A p p^{N L-G-F}$ mice after pioglitazone treatment, although the two groups were clearly separated by TSPO-PET and CD68 immunohistochemistry. Thus, the TSPO-PET signal has a close association with disease-associated microglial activation, which also fits to the strong correlations between CD68 and TSPO-PET reported in Trem2-deficient APPPS1 mice ${ }^{11}$.

PPARy receptor agonists represent a rather unspecific drug since PPARy is involved in various pathways in addition of peroxisome activation, notably including glucose metabolism and insulin sensitization ${ }^{33}$. We selected pioglitazone for immunomodulation of microglial activity in $A D$ mouse models as the effects of this drug are well understood. Nonetheless, more specific drugs like NLRP3 regulators ${ }^{34}$ could enable a more direct targeting of the inflammasome in neurodegenerative diseases. Optimization of immunomodulator strategies could potentially improve their effectiveness and reduce their side effects, whereupon our present TSPO-PET imaging paradigm could be readily transferable to other drugs, so long as they target activated microglia. Ultimately, specific radioligands for different microglia phenotypes ${ }^{35}$ could enhance monitoring of immunomodulation in vivo.

\section{Conclusion}

TSPO-PET serves as a sensitive biomarker for in vivo monitoring of immunomodulation in mouse AD models. Pre-therapeutic assessment of microglial activation in individual mice predicted their response to immunomodulation therapy, indicating that a biomarker of microglial activation could serve for responder stratification. There were pronounced sex differences in the responses to PPARy stimulation effects in vivo. The observed heterogeneity of treatment responses in mice with equal genetic background calls for special consideration in the design of biomarker studies assessing effects of immunomodulation on microglial activation in translational trials in AD patients. 


\section{Material and Methods}

\section{Study design}

All experiments were performed in compliance with the National Guidelines for Animal Protection, Germany, with approval of the local animal care committee of the Government of Oberbayern (Regierung Oberbayern) and overseen by a veterinarian. The experiments also complied with the ARRIVE guidelines and were carried out in accordance with the U.K. Animals (Scientific Procedures) Act, 1986 and associated guidelines, EU Directive 2010/63/EU for animal experiments. The chronic treatment study was performed in two different $A \beta$ mouse models and a longitudinal PET imaging design was applied in both cohorts. Female PS2APP and wild-type mice had their baseline assessment at eight months of age and had follow-up PET imaging at 9.5, 11.5 and 13 months of age. Female and male $A p p^{N L-G-F}$ mice had their baseline assessment at five months of age and received follow-up PET imaging at 7.5 and 10 months of age. Cage randomization to pioglitazone treatment or control chow (vehicle) groups was initiated after the baseline PET scans, and treatments continued until after the terminal behavioural assessments. After recovering from the final PET scan, mice were transferred to the behavioral facility and rested for one week before initiation of Morris water maze (WM) testing of spatial learning. One week after the behavioural tests, mice were deeply anaesthetized prior to transcardial perfusion and fixation with $4 \%$ paraformaldehyde. We then harvested and processed the brains for immunohistochemical and biochemical analyses (randomized hemispheres). Group comparisons of longitudinal A $\beta$-PET monitoring and detailed $A \beta$ analyses by immunohistochemistry and biochemistry of the same cohort are reported in a separate manuscript ${ }^{16}$. Shared datapoints between both manuscripts are indicated and cited.

\section{Animal Models and Statistical Power Analysis}

The transgenic B6.PS2APP (line B6.152H) is homozygous for human presenilin (PS) 2, the $\mathrm{N} 141$ I mutation, and the human amyloid precursor protein (APP) K670N/M671L mutations ${ }^{36}$. Homozygous B6.PS2APP mice show first appearance of plaques in the cerebral cortex and hippocampus at 5-6 months of age ${ }^{37}$. The knock-in mouse model $A p p^{N L-G-F}$ carries a mutant APP gene encoding the humanized $A \beta$ sequence (G601R, F606Y, and $R 609 H$ ) with three pathogenic mutations, namely Swedish (KM595/596NL), Beyreuther/lberian (I641F), and Arctic (E618G). Homozygotic $A p p^{N L-G-F}$ mice progressively exhibit widespread $A \beta$ accumulation from two months of age ${ }^{13}, 38$. Both transgenic models were generated on a C57BI/6 background which also served for wild-type controls. 
Required sample sizes were calculated by G*power (V3.1.9.2, Kiel, Germany), based on assumptions for a type I error $\alpha=0.05$ and a power of 0.8 for group comparisons. A drop-out rate of $10 \%$ per time-point was assumed and a treatment effect causing $5 \%$ change in the PET signal was considered significant. Estimations were based on PET measures in previous investigations with the same mouse models ${ }^{10,39}$. Calculated sample sizes at baseline were $\mathrm{n}=14$ for PS2APP, $\mathrm{n}=8$ for wild-type, and $\mathrm{n}=9$ per sex for $A p p^{N L-G-F}$.

\section{PET Imaging}

For all PET procedures, radiochemistry, data acquisition, and image pre-processing were conducted according to an established, standardized protocol ${ }^{40}$. In brief, ${ }^{18} \mathrm{~F}-\mathrm{GE}-180 \mathrm{TSPO}-$ PET recordings (average dose: $11.5 \pm 2.2 \mathrm{MBq}$ ) with an emission window of $60-90$ min after injection were performed for assessment of cerebral TSPO expression. A $\beta$-PET recordings $\left({ }^{18} \mathrm{~F}\right.$-florbetaben average dose: $\left.12.2 \pm 2.0 \mathrm{MBq}\right)$ with an emission window of 30-60 min after injection were obtained to measure fibrillar cerebral amyloidosis, as reported elsewhere ${ }^{16}$. Isoflurane anesthesia was induced before tracer injection and maintained to the end of the imaging time window. All image analyses were performed using PMOD (version 3.5; PMOD technologies, Basel, Switzerland). Static 30-60 min (Aß-PET) and 60-90 min (TSPO-PET) datasets were co-registered to tracer specific templates (genotype specific) by a manual rigid-body transformation ( $\mathrm{TX}_{\text {rigid }}{ }^{40}$. In the second step, a reader-independent affine coregistration to the tracer-specific template was performed ${ }^{40}$. Here, the initial manually fused images were further normalized by non-linear brain normalization $\left(T_{B N}\right)$ via the PMOD brain normalization tool (equal modality; smoothing by $0.6 \mathrm{~mm}$; nonlinear warping; 16 iterations; frequency cutoff 3; regularization 1.0; no thresholding). The concatenation of $\mathrm{TX}_{\text {rigid }}$ and $\mathrm{TX}_{\mathrm{BN}}$ was then used to obtain optimal resampling with a minimum of interpolation. Normalization of injected radioactivity was performed by the previously validated myocardium correction method ${ }^{41}$ for TSPO-PET and by previously established white matter ${ }^{40}$ (PS2APP) and periaqueductal grey ${ }^{39}\left(A p p^{N L-G-F}\right)$ reference regions for $A \beta-P E T$. TSPO- and A estimates (per time-point and rate of change) deriving from the same neocortical target $\mathrm{VOI}$ $\left(15 \mathrm{~mm}^{3}\right)$ were extracted and compared between treatment and vehicle groups as well as between transgenic mice and wild-type controls by mixed linear models. The TSPO-PET zscore of each individual transgenic mouse at each time-point was calculated by subtraction of the mean TSPO-PET value of vehicle treated age-matched wild-type mice and division by the standard deviation of wild-type mice $\left(z\right.$-score $=\left[\right.$ mean $_{T G}-$ mean $\left.\left._{W T-V e h}\right] / S D_{W T-V e h}\right)$. The zscore deviation per time was defined as a TSPO-PET AUC ${ }^{15}$ and served as an index for microglial activation during the observation time period. For the association analysis between baseline TSPO-PET and changes of TSPO-PET over time ( $\Delta$ z-score = rate of change), we additionally extracted VOls from the Mirrione atlas ${ }^{42}$ to allow evaluation of multiple brain regions. The large cortex VOI of the atlas was split into motor/sensory, auditory/visual and 
entorhinal/piriform cortices to allow evaluation within compartments of functional similarity. A false discovery rate correction for multiple comparisons was applied.

\section{Water maze}

Two slightly different Morris water maze tasks were applied due to facility changes between the investigation of PS2APP and App ${ }^{N L-G-F}$ cohorts. We used a principal component analysis of the standard read outs of each water maze task to generate a robust read-out for correlation analyses ${ }^{43}$. Thus, one quantitative index of water maze performance per mouse was calculated via dimension reduction and correlated with PET imaging. The experimenter was blind according to the phenotype of the animals. Water maze results were also used as an endpoint in the dedicated manuscript on A $\beta$-PET in both mouse models ${ }^{16}$.

PS2APP and age-matched wild-type mice were subjected to a modified water maze task as described previously ${ }^{15,44-46}$ yielding escape latency, distance to the correct platform and correct choice of the platform as read outs. Mice had to distinguish between two visible platforms, one of which was weighted in such a manner that it would float when the mouse climbed on (correct choice), while the other would sink (wrong choice). The correct platform was always located at the same spot in the maze, while the wrong platform as well as the site from which the mice were released into the maze were varied in a pseudorandom fashion. Visual cues on the walls of the laboratory provided orientation. Trials were terminated if the mouse had failed to reach one of the platforms within $30 \mathrm{sec}$ (error of omission). In this case, or in case of a wrong choice, the experimenter placed the mouse on the correct platform. After a three-day handling period, water maze training was performed on five consecutive days, with five trials per day, which were conducted 2-4 minutes apart. Memory performance was assessed by measuring the escape latency at each day of training and by the travelled distance at the last training day. For measuring escape latency, we calculated the summed average time of all trials from the start point to attaining one of the platforms. On the sixth day, the correct platform was placed in the opposite quadrant of the maze to confirm that the mice indeed used spatial cues rather than rule-based learning to find it. Trials were filmed with a video camera and the swimming trace was extracted using custom written LabView software (National Instruments).

$A p p^{N L-G-F}$ mice and 14 age- and sex-matched wild-type mice underwent a common Morris water maze test, which was performed according to a standard protocol with small adjustments ${ }^{47}$ as previously described ${ }^{39}$. In brief, the first day was used for acclimatization with a visible platform (five minutes per mouse). The mice then underwent five training days where each mouse had to perform four trials per day with the platform visible at the first training day and the platform hidden under water for all other training days. The test day was set by only one trial with complete removal of the platform. The maximum trial length on all 
training and test days was set to a maximum of 70 seconds. The video tracking software EthoVision ${ }^{\circledR}$ XT (Noldus) was used for analyses of escape latency, the platform frequency and attendance in the platform quadrant at the probe trial.

The principal component of the water maze test scores was extracted from three spatial learning readouts (PS2APP: escape latency, distance, platform choice; $A p p^{N L-G-F}$ : escape latency, frequency to platform, time spent in platform quadrant) using SPSS 26 statistics (IBM Deutschland GmbH, Ehningen, Germany). Prior to the PCA, the linear relationship of the data was tested by a correlation matrix and items with a correlation coefficient $<0.3$ were discarded. The Kaiser-Meyer-Olkin (KMO) measure and Bartlett's test of sphericity were used to test for sampling adequacy and suitability for data reduction. Components with an Eigenvalue $>1.0$ were extracted and a varimax rotation was selected.

\section{Immunohistochemistry}

Iba-1 and CD68 immunohistochemistry was performed as described previously ${ }^{39,48}$ and the group comparisons between treatment and vehicle groups are reported in the accompanying manuscript ${ }^{16}$. Correlation analyses were performed between TSPO-PET and Iba-1/CD68 quantitation. $\mathrm{n}=4-5$ PS2APP and $A p p^{N L-G-F}$ mice per treatment and vehicle groups with a successful TSPO-PET scan prior to immunohistochemistry were subjected to this analysis. In brief, we performed a standard free-floating immunofluorescence protocol with cortex areas matching the PET brain regions of interest. As previously described, perfusion-fixed $50-\mu \mathrm{m}$ thick brain sections were rinsed either overnight or for $48 \mathrm{~h}$ in PBS with $0.2 \%$ Triton X-100 containing one of the following primary antibodies: rabbit monoclonal lba-1 (1:500. Wako: 1919741), or rat monoclonal CD68 (1:500. Bio-rad: MCA1857). After washing in PBS, sections were then incubated in a combination of three secondary antibodies (Alexa 488 goat antirabbit, Alexa 594 goat anti-mouse). A detailed analysis of A $\beta$-plaques (methoxy-X04 and NAB223) of this cohort is reported in the accompanying manuscript ${ }^{16}$.

\section{Statistics}

Group differences (i.e. between treatment groups or sexes) in TSPO-PET trajectories over time were determined using linear mixed models using the Imer package in the $\mathrm{R}$ statistical software, including a random intercept. Note that we selected models including either linear or quadratic time effects based on best model fit (i.e. lower Akaike Information Criterion for better model fit).

Association analyses were performed between PET, water maze, and immunohistochemistry scores. Pearson's coefficient of correlation (R) was calculated after confirming normal distribution by a Kolmogorov-Smirnow test. Correlation analysis was performed between TSPO-PET baseline (z-score) and the rate of change of TSPO-PET ( $\Delta$ z-score). This 
analysis was performed in the cortical target region and in a separate analysis of the full Mirrione atlas set of VOls ${ }^{42}$. False discovery rate correction was applied for the multi-region analysis. The rate of change of TSPO-PET ( $\Delta$ z-score) was correlated with the principal component of the water maze task to investigate potential associations of the PPARY stimulation treatment effect with spatial learning performance. The index of microglial activity during a certain time-period $(A \cup C)$ was correlated with the $A \beta-P E T$ rate of change to test the hypothesis of $A \beta$ removal by activated microglia ${ }^{18}$. Immunohistochemistry quantification (Iba1 and CD68) in the cortex was correlated with the cortical TSPO-PET signal of the terminal time-point. 


\section{References}

1. Heneka MT, Carson MJ, Khoury JE, Landreth GE, Brosseron F, Feinstein DL et al. Neuroinflammation in Alzheimer's disease. The Lancet Neurology 2015; 14(4): 388-405.

2. Fakhoury M. Microglia and Astrocytes in Alzheimer's Disease: Implications for Therapy. Curr Neuropharmacol 2018; 16(5): 508-518.

3. Ahmad MH, Fatima M, Mondal AC. Influence of microglia and astrocyte activation in the neuroinflammatory pathogenesis of Alzheimer's disease: Rational insights for the therapeutic approaches. J Clin Neurosci 2019; 59: 6-11.

4. Zou C, Shi Y, Ohli J, Schuller U, Dorostkar MM, Herms J. Neuroinflammation impairs adaptive structural plasticity of dendritic spines in a preclinical model of Alzheimer's disease. Acta neuropathologica 2016; 131(2): 235-246.

5. Mandrekar-Colucci S, Karlo JC, Landreth GE. Mechanisms underlying the rapid peroxisome proliferator-activated receptor-gamma-mediated amyloid clearance and reversal of cognitive deficits in a murine model of Alzheimer's disease. J Neurosci 2012; 32(30): 10117-10128.

6. Burns DK, Chiang C, Welsh-Bohmer KA, Brannan SK, Culp M, O'Neil J et al. The TOMMORROW study: Design of an Alzheimer's disease delay-of-onset clinical trial. Alzheimers Dement $(N Y)$ 2019; 5: 661-670.

7. Scott G, Zetterberg H, Jolly A, Cole JH, De Simoni S, Jenkins PO et al. Minocycline reduces chronic microglial activation after brain trauma but increases neurodegeneration. Brain 2018; 141(2): 459-471.

8. Wolf BJ, Brackhan M, Bascunana P, Leiter I, Langer BLN, Ross TL et al. TSPO PET Identifies Different Anti-inflammatory Minocycline Treatment Response in Two Rodent Models of Epileptogenesis. Neurotherapeutics 2020.

9. James ML, Belichenko NP, Shuhendler AJ, Hoehne A, Andrews LE, Condon C et al. [(18)F]GE180 PET Detects Reduced Microglia Activation After LM11A-31 Therapy in a Mouse Model of Alzheimer's Disease. Theranostics 2017; 7(6): 1422-1436.

10. Brendel M, Probst F, Jaworska A, Overhoff F, Korzhova V, Albert NL et al. Glial Activation and Glucose Metabolism in a Transgenic Amyloid Mouse Model: A Triple-Tracer PET Study. I Nucl Med 2016; 57(6): 954-960.

11. Parhizkar S, Arzberger T, Brendel M, Kleinberger G, Deussing M, Focke $\mathrm{C}$ et al. Loss of TREM2 function increases amyloid seeding but reduces plaque-associated ApoE. Nature neuroscience 2019; 22(2): 191-204.

12. Biechele G, Franzmeier N, Blume $\mathrm{T}$, Ewers $\mathrm{M}$, Medina-Luque J, Eckenweber $\mathrm{F}$ et al. Glial activation is moderated by sex in response to amyloidosis but not to tau pathology in mouse models of neurodegenerative diseases. J Neuroinflammation 2020; In press. 
13. Saito T, Matsuba Y, Mihira N, Takano J, Nilsson P, Itohara S et al. Single App knock-in mouse models of Alzheimer's disease. Nature neuroscience 2014; 17(5): 661-663.

14. Blume T, Focke C, Peters F, Deussing M, Albert NL, Lindner S et al. Microglial response to increasing amyloid load saturates with aging: a longitudinal dual tracer in vivo muPET-study. Journal of neuroinflammation 2018; 15(1): 307.

15. Focke $\mathrm{C}$, Blume $\mathrm{T}$, Zott B, Shi $\mathrm{Y}$, Deussing $\mathrm{M}$, Peters $\mathrm{F}$ et al. Early and Longitudinal Microglial Activation but Not Amyloid Accumulation Predicts Cognitive Outcome in PS2APP Mice. Journal of nuclear medicine : official publication, Society of Nuclear Medicine 2019; 60(4): 548-554.

16. Blume T, Deussing M, Biechele G, Peters F, Zott B, Schmidt C et al. Chronic PPARy Stimulation Shifts Amyloidosis to Higher Fibrillarity but Improves Cognition. bioRxiv 2021.

17. Biechele G, Sebastian Monasor L, Wind K, Blume T, Parhizkar S, Arzberger T et al. Glitter in the Darkness? Non-fibrillar beta-amyloid Plaque Components Significantly Impact the betaamyloid PET Signal in Mouse Models of Alzheimer's Disease. J Nucl Med 2021.

18. Ewers M, Biechele G, Suarez-Calvet M, Sacher C, Blume T, Morenas-Rodriguez E et al. Higher CSF STREM2 and microglia activation are associated with slower rates of beta-amyloid accumulation. EMBO Mol Med 2020; 12(9): e12308.

19. Rapic S, Backes H, Viel T, Kummer MP, Monfared P, Neumaier B et al. Imaging microglial activation and glucose consumption in a mouse model of Alzheimer's disease. Neurobiol Aging 2013; 34(1): 351-354.

20. Dickens AM, Vainio S, Marjamaki P, Johansson J, Lehtiniemi P, Rokka J et al. Detection of microglial activation in an acute model of neuroinflammation using PET and radiotracers 11C(R)-PK11195 and 18F-GE-180. J Nucl Med 2014; 55(3): 466-472.

21. Sridharan S, Raffel J, Nandoskar A, Record C, Brooks DJ, Owen D et al. Confirmation of Specific Binding of the 18-kDa Translocator Protein (TSPO) Radioligand [(18)F]GE-180: a Blocking Study Using XBD173 in Multiple Sclerosis Normal Appearing White and Grey Matter. Mol Imaging Biol 2019; 21(5): 935-944.

22. Cumming P, Burgher B, Patkar O, Breakspear M, Vasdev N, Thomas P et al. Sifting through the surfeit of neuroinflammation tracers. J Cereb Blood Flow Metab 2018; 38(2): 204-224.

23. Heneka MT, Carson MJ, Khoury JE, Landreth GE, Brosseron F, Feinstein DL et al. Neuroinflammation in Alzheimer's disease. The Lancet Neurology 2015; 14(4): 388-405.

24. Biechele G, Wind K, Blume T, Sacher C, Beyer L, Eckenweber F et al. Microglial activation in the right amygdala-entorhinal-hippocampal complex is associated with preserved spatial learning in App(NL-G-F) mice. Neuroimage 2020; 230: 117707. 
25. Dorostkar MM, Burgold S, Filser S, Barghorn S, Schmidt B, Anumala UR et al. Immunotherapy alleviates amyloid-associated synaptic pathology in an Alzheimer's disease mouse model. Brain 2014; 137(Pt 12): 3319-3326.

26. Seok H, Lee M, Shin E, Yun MR, Lee YH, Moon JH et al. Low-dose pioglitazone can ameliorate learning and memory impairment in a mouse model of dementia by increasing LRP1 expression in the hippocampus. Sci Rep 2019; 9(1): 4414.

27. Pike CJ. Sex and the development of Alzheimer's disease. J Neurosci Res 2017; 95(1-2): 671680.

28. Jack CR, Jr., Bennett DA, Blennow K, Carrillo MC, Dunn B, Haeberlein SB et al. NIA-AA Research Framework: Toward a biological definition of Alzheimer's disease. Alzheimers Dement 2018; 14(4): 535-562.

29. Bateman RJ, Blennow K, Doody R, Hendrix S, Lovestone S, Salloway $\mathrm{S}$ et al. Plasma Biomarkers of AD Emerging as Essential Tools for Drug Development: An EU/US CTAD Task Force Report. J Prev Alzheimers Dis 2019; 6(3): 169-173.

30. Saito T, Mihira N, Matsuba Y, Sasaguri H, Hashimoto S, Narasimhan S et al. Humanization of the entire murine Mapt gene provides a murine model of pathological human tau propagation. J Biol Chem 2019; 294(34): 12754-12765.

31. Cohen RM, Rezai-Zadeh K, Weitz TM, Rentsendorj A, Gate D, Spivak I et al. A transgenic Alzheimer rat with plaques, tau pathology, behavioral impairment, oligomeric abeta, and frank neuronal loss. J Neurosci 2013; 33(15): 6245-6256.

32. He Z, Guo JL, McBride JD, Narasimhan S, Kim H, Changolkar L et al. Amyloid-beta plaques enhance Alzheimer's brain tau-seeded pathologies by facilitating neuritic plaque tau aggregation. Nat Med 2018; 24(1): 29-38.

33. Tyagi S, Gupta P, Saini AS, Kaushal C, Sharma S. The peroxisome proliferator-activated receptor: A family of nuclear receptors role in various diseases. I Adv Pharm Technol Res 2011; 2(4): 236-240.

34. Swanson KV, Deng M, Ting JP. The NLRP3 inflammasome: molecular activation and regulation to therapeutics. Nat Rev Immunol 2019; 19(8): 477-489.

35. Götzl JK, Brendel M, Werner G, Parhizkar S, Sebastian Monasor L, Kleinberger G et al. Opposite microglial activation stages upon loss of PGRN or TREM2 result in reduced cerebral glucose metabolism. EMBO Molecular Medicine 2019: e9711.

36. Richards JG, Higgins GA, Ouagazzal AM, Ozmen L, Kew JN, Bohrmann B et al. PS2APP transgenic mice, coexpressing hPS2mut and hAPPswe, show age-related cognitive deficits 
associated with discrete brain amyloid deposition and inflammation. J Neurosci 2003; 23(26): 8989-9003.

37. Ozmen L, Albientz A, Czech C, Jacobsen H. Expression of transgenic APP mRNA is the key determinant for beta-amyloid deposition in PS2APP transgenic mice. Neuro-degenerative diseases 2009; 6(1-2): 29-36.

38. Masuda A, Kobayashi Y, Kogo N, Saito T, Saido TC, Itohara S. Cognitive deficits in single App knock-in mouse models. Neurobiol Learn Mem 2016; 135: 73-82.

39. Sacher C, Blume T, Beyer L, Peters F, Eckenweber F, Sgobio C et al. Longitudinal PET Monitoring of Amyloidosis and Microglial Activation in a Second-Generation Amyloid-beta Mouse Model. J Nucl Med 2019; 60(12): 1787-1793.

40. Overhoff F, Brendel M, Jaworska A, Korzhova V, Delker A, Probst F et al. Automated Spatial Brain Normalization and Hindbrain White Matter Reference Tissue Give Improved [(18)F]Florbetaben PET Quantitation in Alzheimer's Model Mice. Frontiers in neuroscience 2016; 10: 45.

41. Deussing M, Blume T, Vomacka L, Mahler C, Focke C, Todica A et al. Coupling between physiological TSPO expression in brain and myocardium allows stabilization of late-phase cerebral [(18)F]GE180 PET quantification. Neuroimage 2017; 165: 83-91.

42. Schiffer WK, Mirrione MM, Biegon A, Alexoff DL, Patel V, Dewey SL. Serial microPET measures of the metabolic reaction to a microdialysis probe implant. Journal of neuroscience methods 2006; 155(2): 272-284.

43. Biechele G, Wind K, Blume T, Sacher C, Beyer L, Eckenweber F et al. Microglial Activation in the Right Amygdala-Entorhinal-Hippocampal Complex is Associated with Preserved Spatial Learning in App(NL-G-F) mice. Neurolmage 2020: 117707.

44. Sauvage $M$, Brabet $P$, Holsboer F, Bockaert J, Steckler T. Mild deficits in mice lacking pituitary adenylate cyclase-activating polypeptide receptor type 1 (PAC1) performing on memory tasks. Brain Res Mol Brain Res 2000; 84(1-2): 79-89.

45. Busche MA, Kekus $\mathrm{M}$, Adelsberger $\mathrm{H}$, Noda $\mathrm{T}$, Forstl $\mathrm{H}$, Nelken I et al. Rescue of long-range circuit dysfunction in Alzheimer's disease models. Nature neuroscience 2015; 18(11): 16231630 .

46. Keskin AD, Kekus M, Adelsberger H, Neumann U, Shimshek DR, Song B et al. BACE inhibitiondependent repair of Alzheimer's pathophysiology. Proceedings of the National Academy of Sciences of the United States of America 2017; 114(32): 8631-8636.

47. Bromley-Brits K, Deng Y, Song W. Morris water maze test for learning and memory deficits in Alzheimer's disease model mice. J Vis Exp 2011; (53). 
48. Eckenweber F, Medina-Luque J, Blume T, Sacher C, Biechele G, Wind K et al. Longitudinal TSPO expression in tau transgenic P301S mice predicts increased tau accumulation and deteriorated spatial learning. J Neuroinflammation 2020; 17(1): 208.

\section{Acknowledgements}

The study was supported by the FöFoLe Program of the Faculty of Medicine of the Ludwig Maximilian University, Munich (grant to M.B.). This work was funded by the Deutsche Forschungsgemeinschaft (DFG, German Research Foundation) to A.R. and M.B. - project numbers BR4580/1-1/ RO5194/1-1. The work was supported by the Deutsche Forschungsgemeinschaft (DFG, German Research Foundation) under Germany's Excellence Strategy within the framework of the Munich Cluster for Systems Neurology (EXC 2145 SyNergy - ID 390857198). M.B. was supported by the Alzheimer Forschung Initiative e.V (grant number 19063p). We thank Karin Bormann-Giglmaier and Rosel Oos for excellent technical assistance. We thank Christian Haass for excellent support and supervision of the project. We thank Takashi Saito and Takaomi C. Saido for providing the $A p p^{N L-G-F}$ mice. GE Healthcare made GE-180 cassettes available through an early-access model. The authors acknowledge Inglewood Biomedical Editing for professional editing of the manuscript.

\section{Disclosures}

K.B. is an employee of Roche. M.B. received speaker honoraria from GE healthcare, Roche and LMI and is an advisor of LMI. 
A
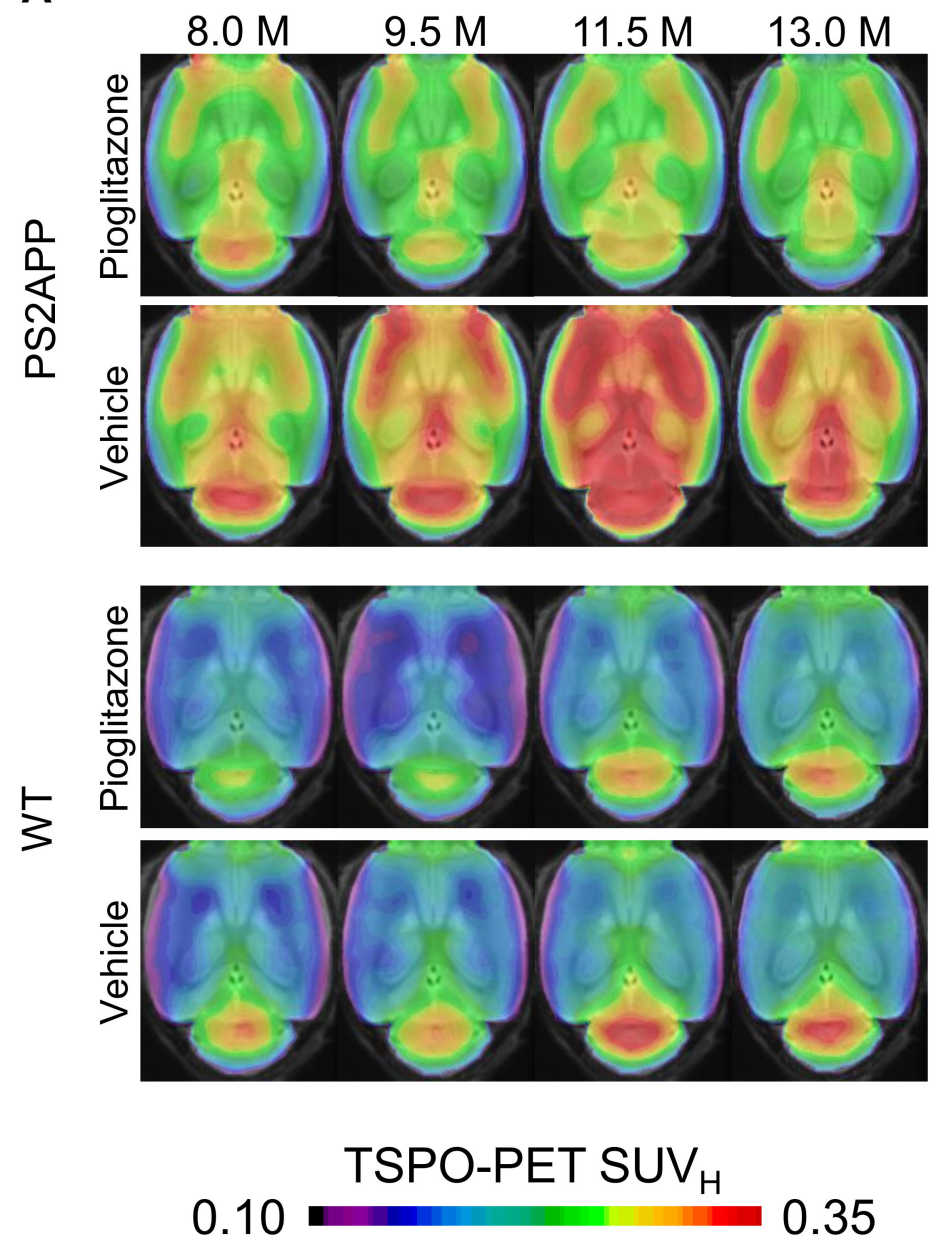

B
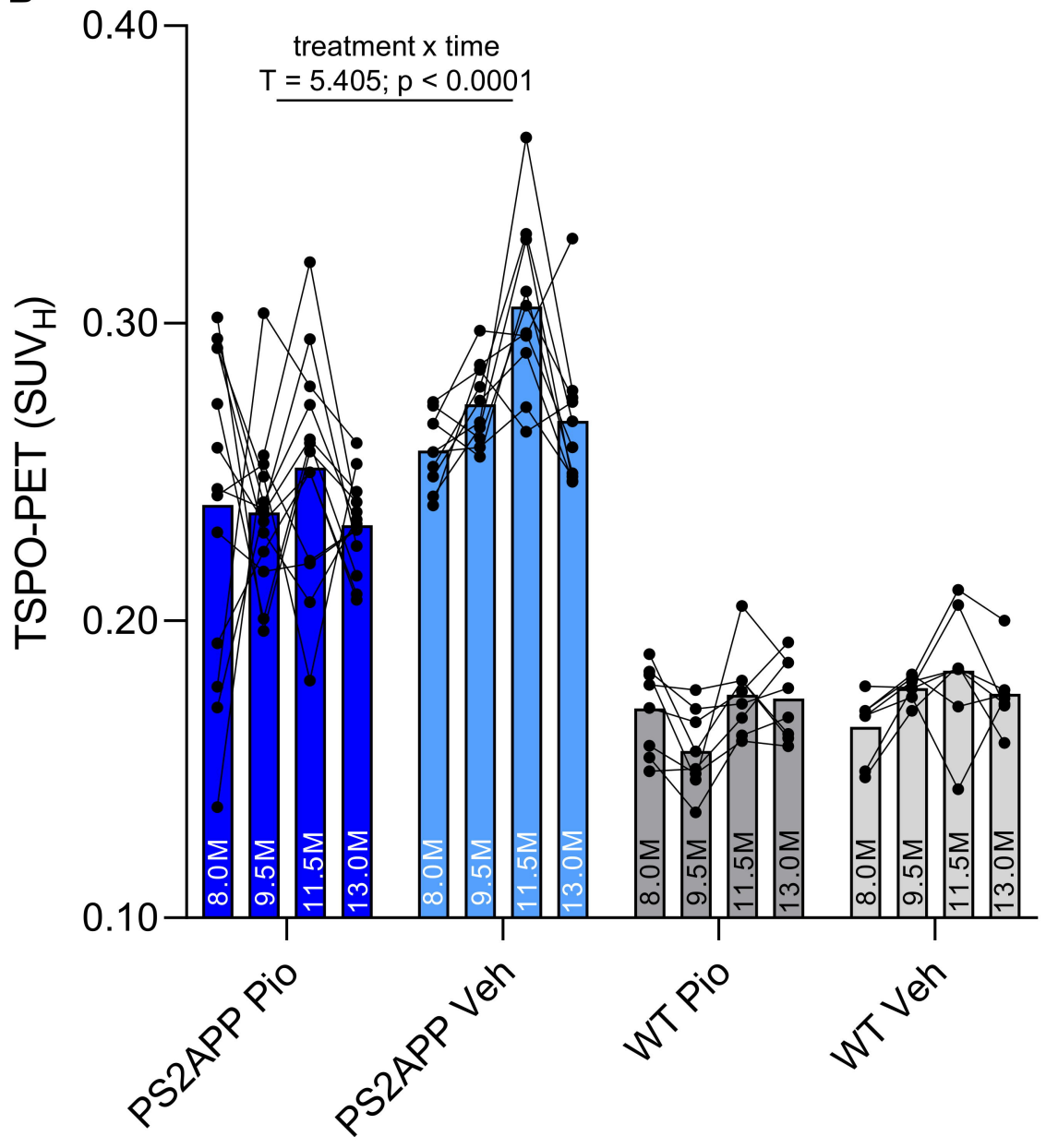
A
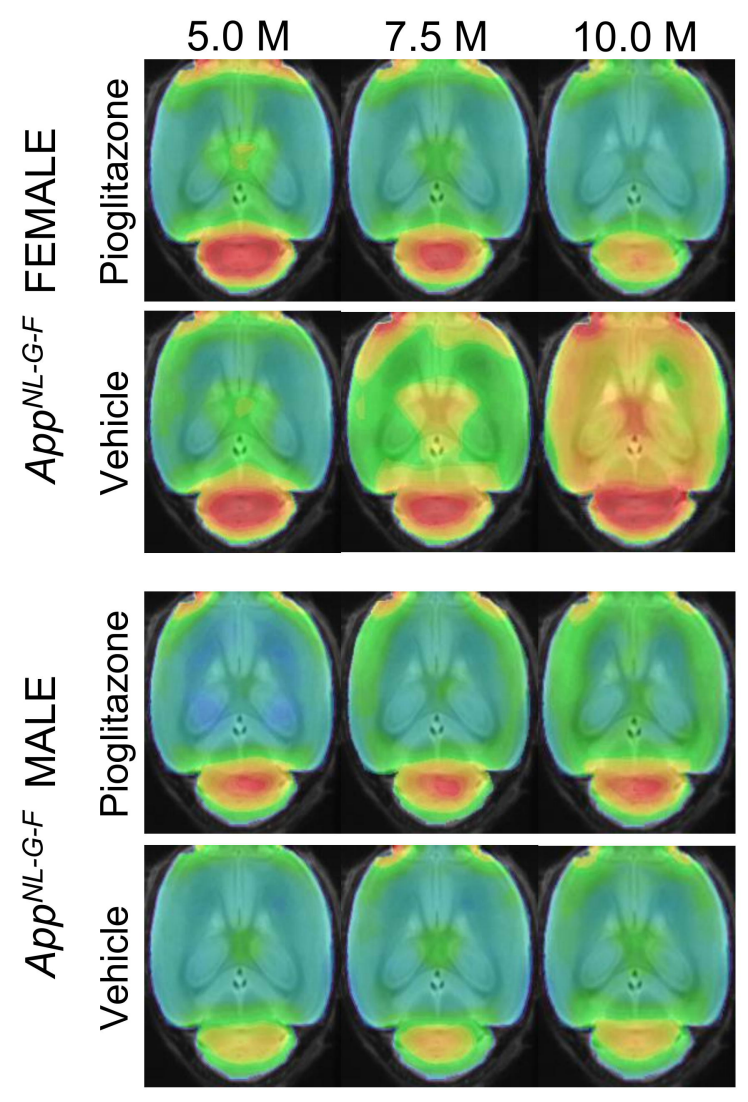

TSPO-PET SUV
B

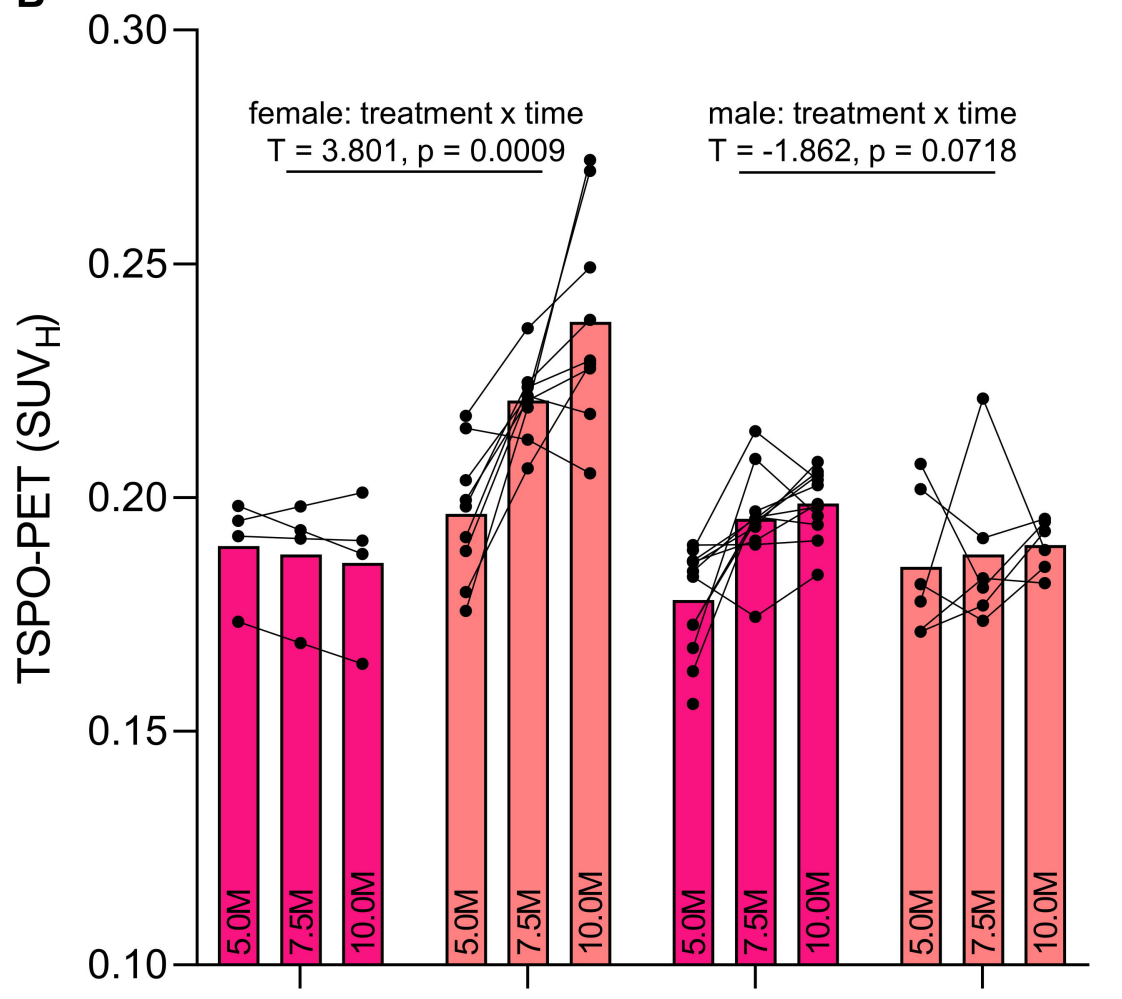

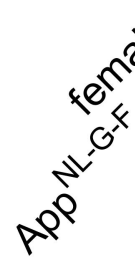
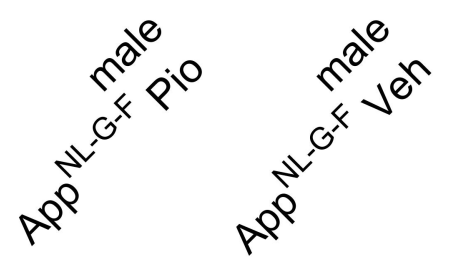


\section{A \\ Pioglitazone}
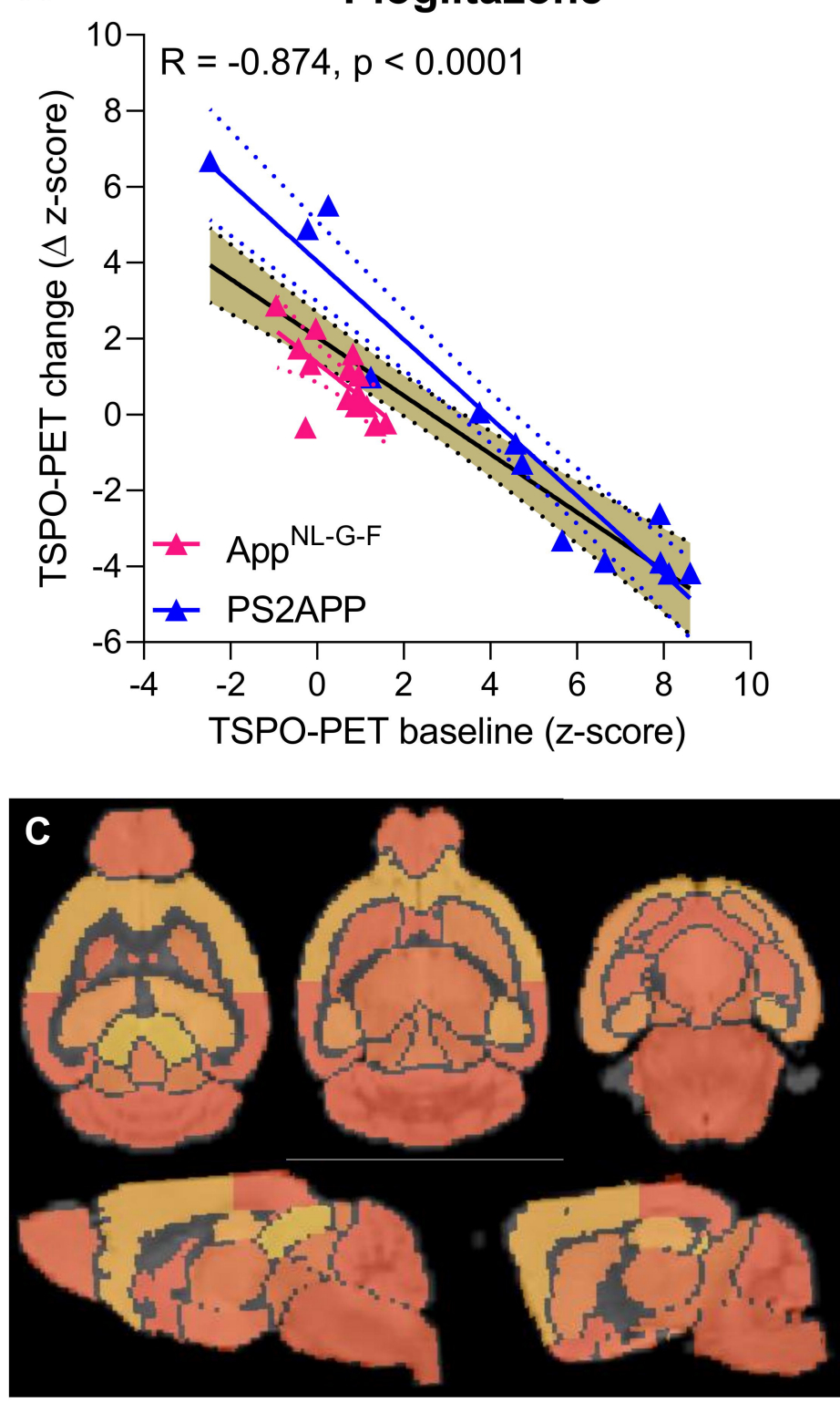

B

Vehicle
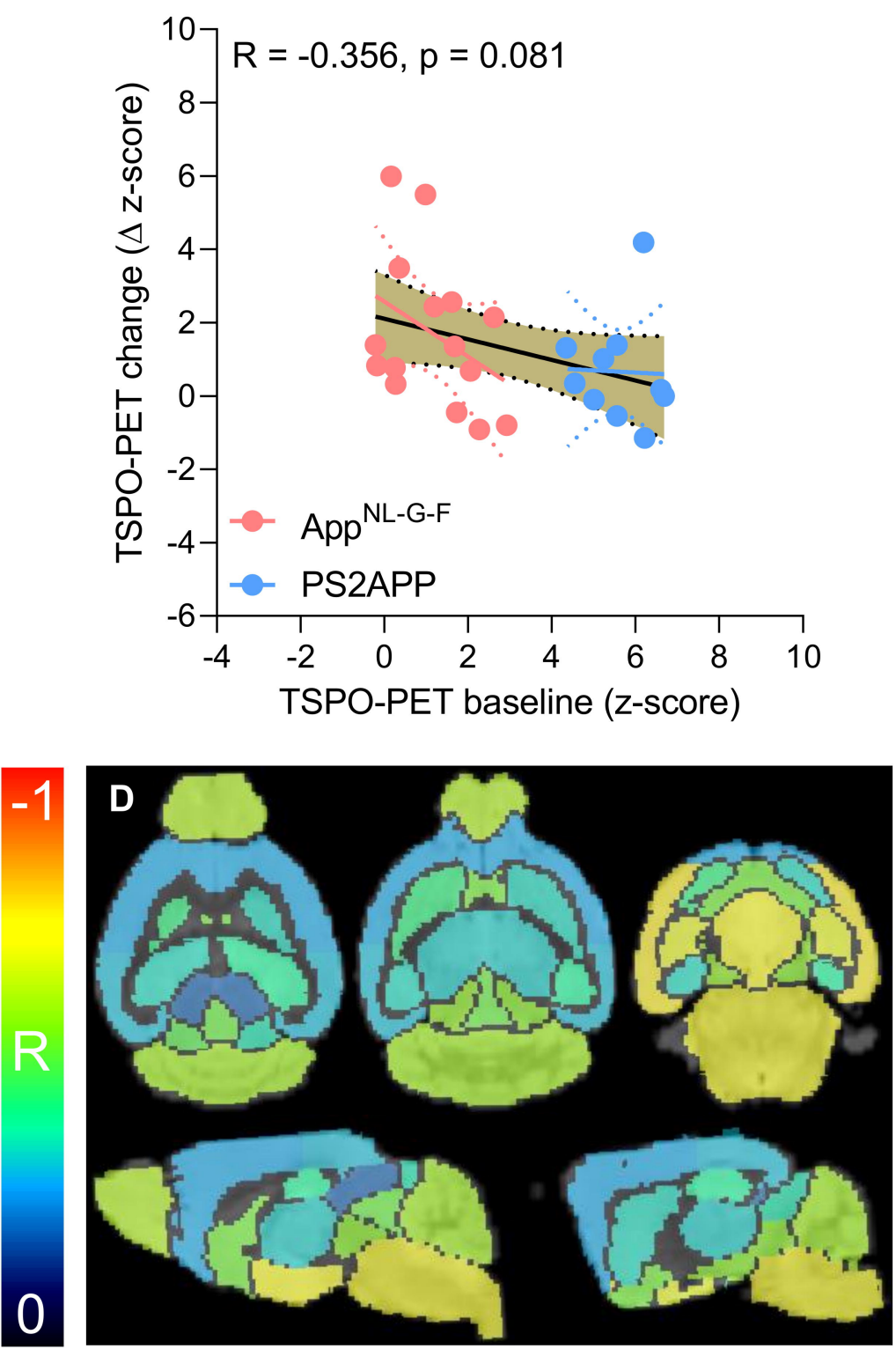

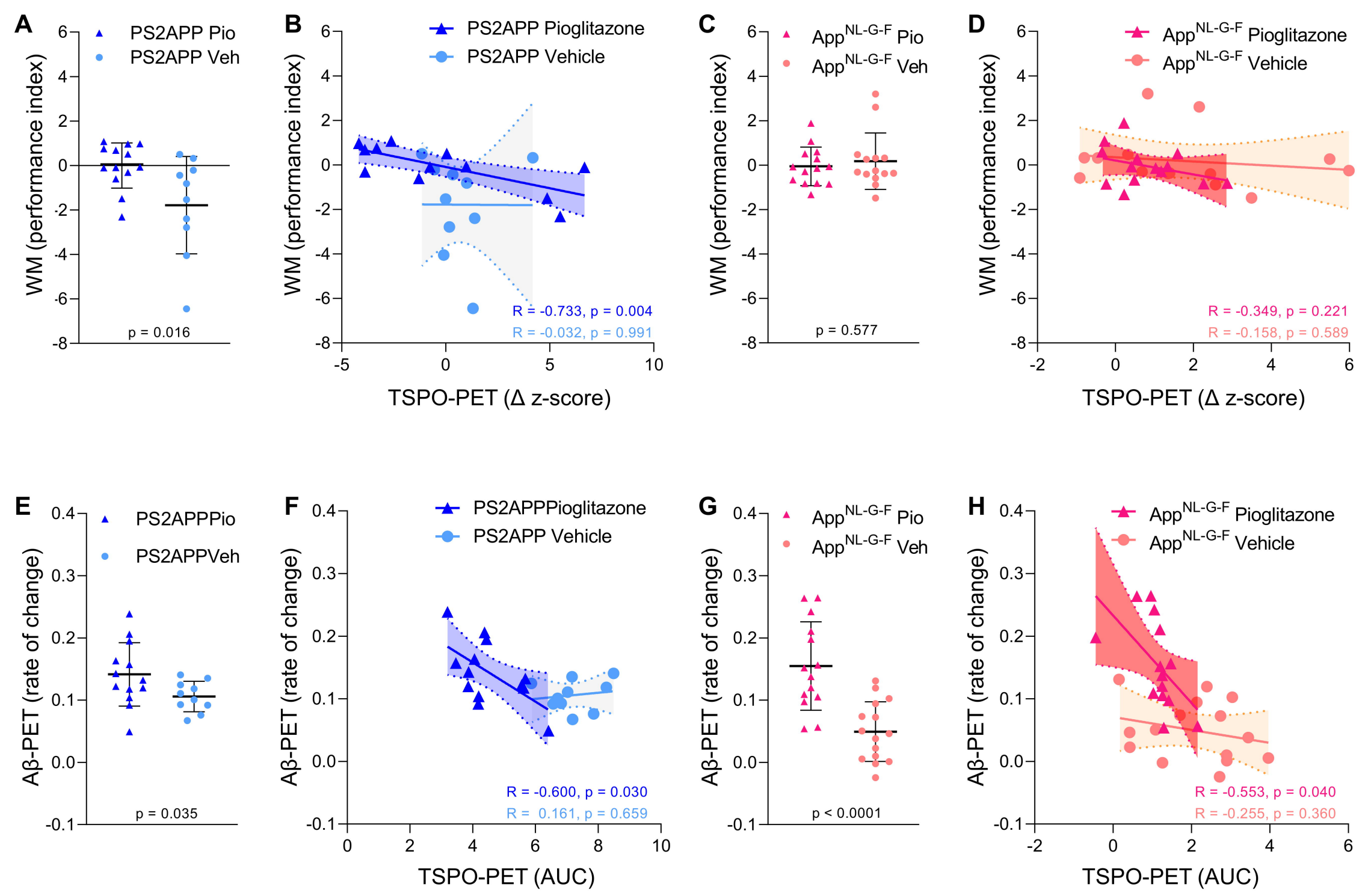
